\title{
FLOATING BODY, ILLUMINATION BODY, AND POLYTOPAL APPROXIMATION
}

\author{
Carsten SchüTt \\ Abstract. Let $K$ be a convex body in $\mathbb{R}^{d}$ and $K_{t}$ its floating bodies. There is a \\ polytope with at most $n$ vertices that satisfies

$$
K_{t} \subset P_{n} \subset K
$$

where

$$
n \leq e^{16 d} \frac{\operatorname{vol}_{d}\left(K \backslash K_{t}\right)}{t \text { vol }_{d}\left(B_{2}^{d}\right)}
$$

Let $K^{t}$ be the illumination bodies of $K$ and $Q_{n}$ a polytope that contains $K$ and has at most $n d-1$-dimensional faces. Then

$$
\operatorname{vol}_{d}\left(K^{t} \backslash K\right) \leq c d^{4} \operatorname{vol}_{d}\left(Q_{n} \backslash K\right)
$$

where

$$
n \leq \frac{c}{d t} \operatorname{vol}_{d}\left(K^{t} \backslash K\right)
$$

1991 Mathematics Subject Classification. 52A22.

This paper was written while the author was visiting the MSRI at Berkeley in the spring of 1996 


\section{INTRODUCTION}

We investigate the approximation of a convex body $K$ in $\mathbb{R}^{d}$ by a polytope. We measure the approximation by the symmetric difference metric. The symmetric difference metric between two convex bodies $K$ and $C$ is

$$
d_{S}(C, K)=\operatorname{vol}_{d}((C \backslash K) \cup(K \backslash C))
$$

We study in particular two questions: How well can a convex body $K$ be approximated by a polytope $P_{n}$ that is contained in $K$ and has at most $n$ vertices and how well can $K$ be approximated by a polytope $Q_{n}$ that contains $K$ and has at most $n$ $d$-1-dimensional faces. Macbeath [Mac] showed that the Euclidean Ball $B_{2}^{d}$ is an extremal case: The approximation for any other convex body is better. We have for the Euclidean ball

$$
c_{1} d \operatorname{vol}_{d}\left(B_{2}^{d}\right) n^{-\frac{2}{d-1}} \leq d_{S}\left(P_{n}, B_{2}^{d}\right) \leq c_{2} d \operatorname{vol}_{d}\left(B_{2}^{d}\right) n^{-\frac{2}{d-1}}
$$

provided that $n \geq\left(c_{3} d\right)^{\frac{d-1}{2}}$. The right hand inequality was first established by Bronshtein and Ivanov $[\mathrm{BI}]$ and Dudley $\left[\mathrm{D}_{1}, \mathrm{D}_{2}\right]$. Gordon, Meyer, and Reisner $\left[\mathrm{GMR}_{1}, \mathrm{GMR}_{2}\right]$ gave a constructive proof for the same inequality. Müller [Mü] showed that random approximation gives the same estimate. Gordon, Reisner, and Schütt [GRS] established the left hand inequality. Gruber [ $\left.\mathrm{Gr}_{2}\right]$ obtained an asymptotic formula. If a convex body $K$ in $\mathbb{R}^{d}$ has a $C^{2}$-boundary with everywhere positive curvature, then

$$
\inf \left\{d_{S}\left(K, P_{n}\right) \mid P_{n} \subset K \text { and } P_{n} \text { has at most n vertices }\right\}
$$

is asymptotically the same as

$$
\frac{1}{2} \operatorname{del}_{d-1}\left(\int_{\partial K} \kappa(x)^{\frac{1}{d+1}} d \mu(x)\right)^{\frac{d+1}{d-1}}\left(\frac{1}{n}\right)^{\frac{2}{d-1}}
$$

where $d e l_{d-1}$ is a constant that is connected with Delone triangulations. In this paper we are not concerned with asymptotic estimates, but with uniform.

$\operatorname{Int}(M)$ denotes the interior of a set $M . H(x, \xi)$ denotes the hyperplane that contains $x$ and is orthogonal to $\xi . H^{+}(x, \xi)$ denotes the halfspace that contains the vector $x-\xi$, and $H^{-}(x, \xi)$ the halfspace containing $x+\xi . e_{i}, i=1, \ldots, d$ denotes the unit vector basis in $\mathbb{R}^{d} .[A, B]$ is the convex hull of the sets $A$ and $B$. The convex floating body $K_{t}$ of a convex body $K$ is the intersection of all halfspaces whose defining hyperplanes cut off a set of volume $t$ from $K$.

The illumination body $K^{t}$ of a convex body $K$ is [W]

$$
\left\{x \in \mathbb{R}^{d} \mid \operatorname{vol}_{d}([x, K] \backslash K) \leq t\right\}
$$

$K^{t}$ is a convex body. It is enough to show this for polytopes. Let $F_{i}$ denote the faces of a polytope $P, \xi_{i}$ the outer normal and $x_{i}$ an element of $F_{i}$. Then we have

$$
\operatorname{vol}_{d}([x, P] \backslash P)=\frac{1}{d} \sum_{i=1}^{n} \max \left\{0,<\xi_{i}, x-x_{i}>\right\} \operatorname{vol}_{d-1}\left(F_{i}\right)
$$




\section{The Floating Body}

Theorem 2.1. Let $K$ be a convex body in $\mathbb{R}^{d}$. Then we have for every $t, 0 \leq t \leq$ $\frac{1}{4} e^{-4}$ vol $_{d}(K)$, that there are $n \in \mathbb{N}$ with

$$
n \leq e^{16 d} \frac{\operatorname{vol}_{d}\left(K \backslash K_{t}\right)}{t \operatorname{vol}_{d}\left(B_{2}^{d}\right)}
$$

and a polytope $P_{n}$ that has $n$ vertices and such that

$$
K_{t} \subset P_{n} \subset K
$$

We want to see what kind of asymptotic estimate we get for bodies with smooth boundary from Theorem 1 . We have $[\mathrm{SW}]$

$$
\begin{aligned}
& \operatorname{vol}_{d}\left(K \backslash K_{t}\right) \sim t^{\frac{2}{d+1}} \frac{1}{2}\left(\frac{d+1}{\operatorname{vol}_{d-1}\left(B_{2}^{d-1}\right)}\right)^{\frac{2}{d+1}} \int_{\partial K} \kappa(x)^{\frac{1}{d+1}} d \mu(x) \\
& \sim t^{\frac{2}{d+1}} d \int_{\partial K} \kappa(x)^{\frac{1}{d+1}} d \mu(x)
\end{aligned}
$$

Since

$$
n \sim d^{\frac{d}{2}} \frac{1}{t} \operatorname{vol}_{d}\left(K \backslash K_{t}\right)
$$

we get

$$
\begin{gathered}
\operatorname{vol}_{d}\left(K \backslash K_{t}\right) \sim d\left(d^{\frac{d}{2}} \frac{1}{n} \operatorname{vol}_{d}\left(K \backslash K_{t}\right)\right)^{\frac{2}{d+1}} \int_{\partial K} \kappa(x)^{\frac{1}{d+1}} d \mu(x) \\
\operatorname{vol}_{d}\left(K \backslash K_{t}\right)^{\frac{d-1}{d+1}} \sim d^{2} n^{-\frac{2}{d+1}} \int_{\partial K} \kappa(x)^{\frac{1}{d+1}} d \mu(x)
\end{gathered}
$$

Thus we get

$$
\operatorname{vol}_{d}\left(K \backslash P_{n}\right) \leq \operatorname{vol}_{d}\left(K \backslash K_{t}\right) \sim d^{2} n^{-\frac{2}{d-1}}\left(\int_{\partial K} \kappa(x)^{\frac{1}{d+1}} d \mu(x)\right)^{\frac{d+1}{d-1}}
$$

In case that $K$ is the Euclidean ball we get

$$
\operatorname{vol}_{d}\left(B_{2}^{d} \backslash P_{n}\right) \leq c d^{2} n^{-\frac{2}{d-1}} \operatorname{vol}_{d}\left(B_{2}^{d}\right)
$$

where $c$ is an absolute constant. If one compares this to the optimal result (1.1) one sees that there is an additional factor $d$.

The volume difference $\operatorname{vol}_{d}(P)-\operatorname{vol}_{d}\left(P_{t}\right)$ for a polytope $P$ is of a much smaller order than for a convex body with smooth boundary. In fact, we have $[\mathrm{S}]$ that it is of the order $t|\ln t|^{d-1}$. In [S] this has been used to get estimates for approximation of convex bodies by polytopes.

The same result as in Theorem 2.1 holds if we fix the number of (d-1)-dimensional faces instead of the number of vertices. This follows from the economic cap covering for floating bodies [BL, Theorem 6]. The constants are not as good as in Theorem 2.1 .

The following lemmata are not new. They have usually been formulated for symmetric, convex bodies $[\mathrm{B}, \mathrm{H}, \mathrm{MP}]$. 
Lemma 2.2. Let $K$ be a convex body in $\mathbb{R}^{d}$ and let $H(c g(K), \xi)$ be the hyperplane passing through the center of gravity $\mathrm{cg}(K)$ of $K$ and being orthogonal to $\xi$. Then we have for all $\xi \in \partial B_{2}^{d}$

(i)

$$
\left(1-\frac{1}{d+1}\right)^{d} \operatorname{vol}_{d}(K) \leq \operatorname{vol}_{d}\left(K \cap H^{+}(c g(K), \xi)\right) \leq\left(1-\left(1-\frac{1}{d+1}\right)^{d}\right) \operatorname{vol}_{d}(K)
$$

(ii) for all hyperplanes $H$ in $\mathbb{R}^{d}$ that are parallel to $H(c g(K), \xi)$

$$
\left(1-\frac{1}{d+1}\right)^{d-1} \operatorname{vol}_{d-1}(K \cap H) \leq \operatorname{vol}_{d-1}(K \cap H(c g(K), \xi))
$$

The sequence $\left(1-\frac{1}{d+1}\right)^{d}, d=2,3, \ldots$ is monotonely decreasing. Indeed, by Bernoulli's inequality we have $1-\frac{1}{d} \leq\left(1-\frac{1}{d^{2}}\right)^{d}$, or $\frac{d-1}{d} \leq\left(\frac{d^{2}-1}{d^{2}}\right)^{d}$. Therefore we get $\left(\frac{d}{d+1}\right)^{d} \leq\left(\frac{d-1}{d}\right)^{d-1}$, which implies $\left(1-\frac{1}{d+1}\right)^{d} \leq\left(1-\frac{1}{d}\right)^{d-1}$.

Therefore we get for the inequalities (i)

$$
\frac{1}{e} \operatorname{vol}_{d}(K) \leq \operatorname{vol}_{d}\left(K \cap H^{+}(c g(K), \xi)\right) \leq\left(1-\frac{1}{e}\right) \operatorname{vol}_{d}(K)
$$

By the above $\left(1+\frac{1}{d}\right)^{d}$ is a monotonely increasing sequence. Thus we get $\left(1+\frac{1}{d}\right)^{d-1}<$ e. For (ii) we get

$$
\operatorname{vol}_{d-1}(K \cap H) \leq e \operatorname{vol}_{d-1}(K \cap H(c g(K), \xi))
$$

Proof. (i) We can reduce the inequality to the case that $K$ is a cone with a Euclidean ball of dimension $d-1$ as base. To see this we perform a Schwarz symmetrization parallel to $H(c g(K), \xi)$ and denote the symmetrized body by $S(K)$. The Schwarz symmetrization replaces a section parallel to $H(c g(K), \xi)$ by a $d$-1-dimensional Euclidean sphere of the same $d$-1-dimensional volume. This does not change the volume of $K$ and $K \cap H^{+}(c g(K), \xi)$ and the center of gravity $c g(K)$ is still an element of $H(c g(K), \xi)$. Now we consider the cone

$$
[z, S(K) \cap H(c g(K), \xi)]
$$

such that

$$
\operatorname{vol}_{d}([z, S(K) \cap H(c g(K), \xi)])=\operatorname{vol}_{d}\left(K \cap H^{-}(c g(K), \xi)\right)
$$

and such that $z$ is an element of the axis of symmetry of $S(K)$ and of $H^{-}(c g(K), \xi)$. See figure 2.1.

$$
\tilde{K}=\left(K \cap H^{+}(c g(K), \xi)\right) \cup[z, S(K) \cap H(c g(K), \xi)]
$$

is a convex set such that $\operatorname{vol}_{d}(K)=\operatorname{vol}_{d}(\tilde{K})$ and such that the center of gravity $c g(\tilde{K})$ of $\tilde{K}$ is contained in $[z, S(K) \cap H(c g(K), \xi)]$. Thus 
We apply a similar argument to the set $S(K) \cap H^{+}(c g(K), \xi)$ and show that we may assume that $S(K)$ is a cone with $z$ as its vertex. Thus we may assume that

$$
K=\left[(0, \ldots, 0,1),\left\{\left.\left(x_{1}, \ldots, x_{d-1}, 0\right)\left|\sum_{i=1}^{d-1}\right| x_{i}\right|^{2} \leq 1\right\}\right] \quad \text { and } \quad \xi=(0, \ldots, 0,1)
$$

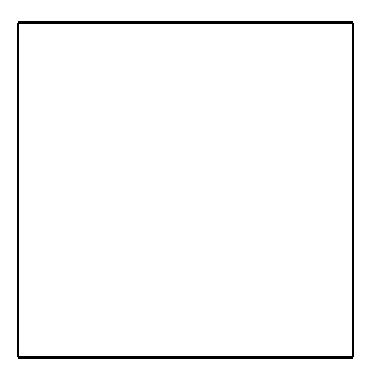

Then

$$
\operatorname{vol}_{d}(K)=\frac{1}{d} \operatorname{vol}_{d-1}\left(B_{2}^{d-1}\right)
$$

and

$$
\frac{1}{v^{\prime} l_{d}(K)} \int_{K} x_{d} d x_{d}=d \int_{0}^{1} t(1-t)^{d-1} d t=d \int_{0}^{1}(1-s) s^{d-1} d s=\frac{1}{d+1}
$$

We obtain that

$$
\operatorname{vol}_{d}\left(K \cap H^{-}(c g(K),(0, \ldots, 0,1))=\left(1-\frac{1}{d+1}\right)^{d} \operatorname{vol}_{d}(K)\right.
$$

(ii) Let $H$ be a hyperplane that is parallel to $H(c g(K), \xi)$ and such that $\operatorname{vol}_{d-1}(K \cap$ $H)>\operatorname{vol}_{d-1}(K \cap H(c g(K), \xi))$. Otherwise there is nothing to prove. We apply a Schwarz symmetrization parallel to $H(c g(K), \xi)$ to $K$. The symmetrized body is denoted by $S(K)$. Let $z$ be the element of the axis of symmetry of $S(K)$ such that

$$
[z, S(K) \cap H] \cap H(c g(K), \xi)=S(K) \cap H(c g(K), \xi)
$$

Since $\operatorname{vol}_{d-1}(K \cap H)>\operatorname{vol}_{d-1}(K \cap H(c g(K), \xi))$ there is such a $z$. We may assume that $H^{+}(c g(K), \xi)$ is the half space containing $z$. Then we have

$$
\begin{aligned}
& {[z, S(K) \cap H] \cap H^{-}(c g(K), \xi) \subset S(K) \cap H^{-}(c g(K), \xi)} \\
& {[z, S(K) \cap H] \cap H^{+}(c g(K), \xi) \supset S(K) \cap H^{+}(c g(K), \xi)}
\end{aligned}
$$

Therefore we have that

$$
c g([z, S(K) \cap H]) \in H^{+}(c g(K), \xi)
$$

Therefore, if $h_{c g}$ denotes the distance of $z$ to $H(c g(K), \xi)$ and $h$ the distance of $z$ to $H$, we get as in the proof of (i) that

$$
h_{c g} \geq h\left(1-\frac{1}{d+1}\right)
$$

Thus we get

$$
\begin{gathered}
\operatorname{vol}_{d-1}(K \cap H(c g(K), \xi))=\operatorname{vol}_{d-1}(S(K) \cap H(c g(K), \xi)) \geq \\
\left(1-\frac{1}{d+1}\right)^{d-1} \operatorname{vol}_{d-1}(S(K) \cap H)=\left(1-\frac{1}{d+1}\right)^{d-1} \operatorname{vol}_{d-1}(K \cap H)
\end{gathered}
$$


Lemma 2.3. Let $K$ be a convex body in $\mathbb{R}^{d}$ and let $\Theta(\xi)$ be the infimum of all numbers $t, 0<t$, such that

$$
\operatorname{vol}_{d-1}(K \cap H(c g(K), \xi)) \geq \operatorname{evol}_{d-1}(K \cap H(c g(K)+t \xi, \xi))
$$

Then we have

$$
\frac{1}{2 e^{3}} \operatorname{vol}_{d}(K) \leq \Theta(\xi) \operatorname{vol}_{d-1}(K \cap H(c g(K), \xi)) \leq e \operatorname{vol}_{d}(K)
$$

Proof. The right hand inequality follows from Fubini's theorem and Brunn-Minkowski's theorem. Now we verify the left hand inequality. We consider first the case that we have for $t, t>\Theta(\xi)$,

$$
K \cap H(c g(K)+t \xi, \xi)=\emptyset
$$

Then we have by (2.1) and (2.2)

$$
\begin{aligned}
\frac{1}{e} \operatorname{vol}_{d}(K) & \leq \operatorname{vol}_{d}\left(K \cap H^{+}(c g(K), \xi)\right) \\
& =\int_{0}^{\Theta(\xi)} \operatorname{vol}_{d-1}(K \cap H(c g(K)+t \xi, \xi)) d t \leq e \Theta(\xi) \operatorname{vol}_{d-1}(H(c g(K), \xi))
\end{aligned}
$$

If for some $t, t>\Theta(\xi)$, we have $K \cap H(c g(K)+t \xi, \xi) \neq \emptyset$ then we have

$$
\operatorname{vol}_{d-1}(K \cap H(c g(K), \xi))=e \operatorname{vol}_{d-1}(K \cap H(c g(K)+\Theta(\xi) \xi, \xi))
$$

We perform a Schwarz symmetrization parallel to $H(c g(K), \xi)$. We consider the cone

$$
[z, S(K) \cap H(c g(K), \xi)]
$$

such that $z$ is an element of the axis of symmetry of $S(K)$ and such that

$$
[z, S(K) \cap H(c g(K), \xi)] \cap H(c g(K)+\Theta(\xi) \xi, \xi)=S(K) \cap H(c g(K)+\Theta(\xi) \xi, \xi)
$$

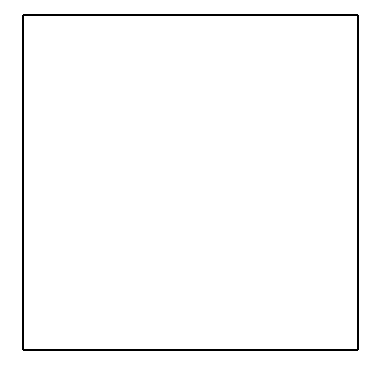

Let $H^{+}(c g(K), \xi)$ and $H^{+}(c g(K)+\Theta(\xi) \xi, \xi)$ be the half spaces that contain $z$. Then we get by convexity

$$
\begin{aligned}
{[z, S(K) \cap H(c g(K), \xi)] } & \cap H^{+}(c g(K)+\Theta(\xi) \xi, \xi) \\
& \supset S(K) \cap H^{+}(c g(K)+\Theta(\xi) \xi, \xi)
\end{aligned}
$$

We get by $(2.1)$ 
FLOATING BODY, ILLUMINATION BODY, AND POLYTOPAL APPROXIMATION

$$
\begin{aligned}
& \operatorname{vol}_{d}\left(K \cap H^{+}(c g(K), \xi) \cap H^{-}\right.(c g(K)+\Theta(\xi) \xi, \xi))+ \\
& \operatorname{vol}_{d}\left(K \cap H^{+}(c g(K)+\Theta(\xi) \xi, \xi)\right)= \\
& \operatorname{vol}_{d}\left(S(K) \cap H^{+}(c g(K), \xi) \cap\right.\left.H^{-}(c g(K)+\Theta(\xi) \xi, \xi)\right)+ \\
& \operatorname{vol}_{d}\left(S(K) \cap H^{+}(\operatorname{cg}(K)+\Theta(\xi) \xi, \xi)\right)
\end{aligned}
$$

By the hypothesis of the lemma we have for all $s$ with $0 \leq s \leq \Theta(\xi)$

$$
\operatorname{vol}_{d-1}(K \cap H(c g(K), \xi)) \leq e \operatorname{vol}_{d-1}(K \cap H(c g(K)+s \xi, \xi))
$$

Using this and (2.2) we estimate the first summand. The second summand is estimated by using (2.3). Thus the above expression is not greater than

$$
\begin{aligned}
& e^{2} \operatorname{vol}_{d}([z, S(K) \cap H(c g(K), \xi)]\left.\cap H^{-}(c g(K)+\Theta(\xi) \xi, \xi)\right)+ \\
& \operatorname{vol}_{d}\left([z, S(K) \cap H(c g(K), \xi)] \cap H^{+}(c g(K)+\Theta(\xi) \xi, \xi)\right)
\end{aligned}
$$

By an elementary computation for the volume of a cone we get that the latter expression is smaller than

$$
2 e^{2} \operatorname{vol}_{d}\left([z, S(K) \cap H(c g(K), \xi)] \cap H^{-}(c g(K)+\Theta(\xi) \xi, \xi)\right)
$$

We use (2.2) again and get that the above expression is smaller than

$$
2 e^{3} \Theta(\xi) \operatorname{vol}_{d-1}(K \cap H(c g(K), \xi))
$$

Lemma 2.4. Let $K$ be a convex body in $\mathbb{R}^{d}$. Then there is a linear transform $T$ with $\operatorname{det}(T)=1$ so that we have for all $\xi \in \partial B_{2}^{d}$

$$
\int_{T(K)}|<x, \xi>|^{2} d x=\frac{1}{d} \int_{T(K)} \sum_{i=1}^{d}\left|<x, e_{i}>\right|^{2} d x
$$

We say that a convex body is in an isotropic position if the linear transform $T$ in Lemma 2.4 can be chosen to be the identity. See $[\mathrm{B}, \mathrm{H}]$.

Proof. We claim that there is a orthogonal transform $U$ such that we have for all $i, j=1, \ldots, d$ with $i \neq j$,

$$
\int_{U(K)}<x, e_{i}><x, e_{j}>d x=0
$$

Clearly, the matrix

$$
\left(\int_{K}<x, e_{i}><x, e_{j}>d x\right)_{i, j=1}^{d}
$$

is symmetric. Therefore there is an orthogonal $d \times d$-matrix $U$ so that

$$
U\left(\int<x, e_{i}><x, e_{j}>d x\right)_{i j=1}^{d} U^{t}
$$


is a diagonal matrix. We have

$$
\begin{aligned}
& U\left(\int_{K}<x, e_{i}><x, e_{j}>d x\right)_{i, j=1}^{d} U^{t}=\left(\int_{K} \sum_{i, j=1}^{d} u_{l, i}<x, e_{i}><x, e_{j}>u_{k, j} d x\right)_{l, k=1}^{d} \\
& =\left(\int_{K}<x, U^{t}\left(e_{l}\right)><x, U^{t}\left(e_{k}\right)>d x\right)_{l, k=1}^{d}=\left(\int_{U(K)}<y, e_{l}><y, e_{k}>d y\right)_{l, k=1}^{d}
\end{aligned}
$$

So the latter matrix is a diagonal matrix. All the diagonal elements are strictly positive. This argument is repeated with a diagonal matrix so that the diagonal elements turn out to be equal. Therefore there is a matrix $T$ with $\operatorname{det} T=1$ such that

$$
\int_{T(K)}<x, e_{i}><x, e_{j}>d x= \begin{cases}0 & \text { if } i \neq j \\ \frac{1}{d} \int_{T(K)} \sum_{j=1}^{d}\left|<x, e_{j}>\right|^{2} d x & \text { if } i=j\end{cases}
$$

From this the lemma follows.

Lemma 2.5. Let $K$ be a convex body in $\mathbb{R}^{d}$ that is in an isotropic position and whose center of gravity is at the origin. Then we have for all $\xi \in \partial B_{2}^{d}$

$\frac{1}{24 e^{10}} \operatorname{vol}_{d}(K)^{3} \leq \operatorname{vol}_{d-1}(K \cap H(c g(K), \xi))^{2} \frac{1}{d} \int_{K} \sum_{i=1}^{d}\left|<x, e_{i}>\right|^{2} d x \leq 6 e^{3} \operatorname{vol}_{d}(K)^{3}$

Proof. By Lemma 2.4 we have for all $\xi \in \partial B_{2}^{d}$

$$
\frac{1}{d} \int_{K} \sum_{i=1}^{d}\left|<x, e_{i}>\right|^{2} d x=\int_{K}|<x, \xi>|^{2} d x
$$

By Fubini's theorem we get that this equals

$$
\int_{-\infty}^{\infty} t^{2} \operatorname{vol}_{d-1}(K \cap H(t \xi, \xi)) d t \geq \int_{0}^{\Theta(\xi)} t^{2} \operatorname{vol}_{d-1}(K \cap H(t \xi, \xi)) d t
$$

where $\Theta(\xi)$ is as defined in Lemma 2.3. By the definition of $\Theta(\xi)$ the above expression is greater than

$$
\frac{1}{e} \operatorname{vol}_{d-1}(K \cap H(c g(K), \xi)) \int_{0}^{\Theta(\xi)} t^{2} d t \geq \frac{1}{3 e} \Theta(\xi)^{3} \operatorname{vol}_{d-1}(K \cap H(c g(K), \xi))
$$

By Lemma 2.3 this is greater than

$$
1 \quad \operatorname{vol}_{d}(K)^{3}
$$


Now we show the right hand inequality. By Lemma 2.4 we have

$$
\begin{gathered}
\frac{1}{d} \int_{K} \sum_{i=1}^{d}\left|<x, e_{i}>\right|^{2} d x=\int_{K}|<x, \xi>|^{2} d x=\int_{-\infty}^{\infty} t^{2} \operatorname{vol}_{d-1}(K \cap H(t \xi, \xi)) d t= \\
\quad \int_{0}^{\Theta(\xi)} t^{2} \operatorname{vol}_{d-1}(K \cap H(t \xi, \xi)) d t+\int_{\Theta(\xi)}^{\infty} t^{2} \operatorname{vol}_{d-1}(K \cap H(t \xi, \xi)) d t+ \\
\int_{\Theta(-\xi)}^{0} t^{2} \operatorname{vol}_{d-1}(K \cap H(t \xi, \xi)) d t+\int_{-\infty}^{\Theta(-\xi)} t^{2} \operatorname{vol}_{d-1}(K \cap H(t \xi, \xi)) d t
\end{gathered}
$$

By (2.2) this is not greater than

$$
\begin{gathered}
\frac{e}{3} \Theta(\xi)^{3} \operatorname{vol}_{d-1}(K \cap H(c g(K), \xi))+\int_{\Theta(\xi)}^{\infty} t^{2} \operatorname{vol}_{d-1}(K \cap H(t \xi, \xi)) d t+ \\
\frac{e}{3} \Theta(-\xi)^{3} \operatorname{vol}_{d-1}(K \cap H(c g(K), \xi))+\int_{-\infty}^{\Theta(-\xi)} t^{2} \operatorname{vol}_{d-1}(K \cap H(t \xi, \xi)) d t
\end{gathered}
$$

The integrals can be estimated by

$$
2 \Theta(\xi)^{3} \operatorname{vol}_{d-1}(K \cap H(c g(K), \xi)) \quad \text { and } \quad 2 \Theta(-\xi)^{3} \operatorname{vol}_{d-1}(K \cap H(c g(K), \xi))
$$

respectively. We treat here only the case $\xi$, the case $-\xi$ is treated in the same way. If the integral equals 0 then there is nothing to show. If the integral does not equal 0 then we have

$$
\operatorname{vol}_{d-1}(K \cap H(c g(K), \xi))=e \operatorname{vol}_{d-1}(K \cap H(c g(K)+\Theta(\xi) \xi, \xi))
$$

We consider the Schwarz symmetrization $S(K)$ of $K$ with respect to the plane $H(c g(K), \xi)$. We consider the cone $C$ that is generated by the Euclidean spheres $S(K) \cap H(c g(K), \xi)$ and $S(K) \cap H(c g(K)+\Theta(\xi) \xi, \xi)$. We have that

$$
S(K) \cap H^{+}(c g(K)+\Theta(\xi) \xi, \xi) \subset C
$$

and that the height of $C$ is equals

$$
\frac{\Theta(\xi)}{1-e^{-\frac{1}{d-1}}}
$$

Since $\left(1+\frac{1}{d-1}\right)^{d-1}<e$ we have $1-e^{-\frac{1}{d-1}}>\frac{1}{d}$. Thus the height of the cone $C$ is less than $d \Theta(\xi)$. Thus we get for all $t$ with $\Theta(\xi) \leq t \leq d \Theta(\xi)$

$$
\operatorname{vol}_{d-1}\left(K \cap H(c g(K)+t \xi, \xi) \leq\left(1-\frac{t}{1 \cap(s)}\right)^{d-1} \operatorname{vol}_{d-1}(K \cap H(c g(K), \xi))\right.
$$


Now we get

$$
\begin{aligned}
\int_{\Theta(\xi)}^{\infty} t^{2} \operatorname{vol}_{d-1}( & K \cap H(t \xi, \xi)) d t \leq \\
& \int_{\Theta(\xi)}^{d \Theta(\xi)} t^{2}\left(1-\frac{t}{d \Theta(\xi)}\right)^{d-1} \operatorname{vol}_{d-1}(K \cap H(c g(K), \xi)) d t \leq \\
& \operatorname{vol}_{d-1}(K \cap H(c g(K), \xi))(d \Theta(\xi))^{3} \int_{0}^{1} s^{2}(1-s)^{d-1} d s= \\
& \operatorname{vol}_{d-1}(K \cap H(c g(K), \xi))(d \Theta(\xi))^{3} \frac{2}{d(d+1)(d+2)} \leq \\
& 2 \operatorname{vol}_{d-1}(K \cap H(c g(K), \xi)) \Theta(\xi)^{3}
\end{aligned}
$$

Therefore we get

$$
\frac{1}{d} \int_{K} \sum_{i=1}^{d}\left|<x, e_{i}>\right|^{2} d x \leq\left(\frac{e}{3}+2\right)\left(\Theta(\xi)^{3}+\Theta(-\xi)^{3}\right) v l_{d-1}(K \cap H(c g(K), \xi))
$$

Now we apply Lemma 2.3 and get

$$
2\left(\frac{e}{3}+2\right) e^{3} \frac{\operatorname{vol}_{d}(K)^{3}}{\operatorname{vol}_{d-1}(K \cap H(c g(K), \xi))^{2}}
$$

Lemma 2.6. Let $K$ be a convex body in $\mathbb{R}^{d}$ such that the origin is an element of $K$. Then we have

$$
\frac{1}{d} \int_{K} \sum_{i=1}^{d}\left|<x, e_{i}>\right|^{2} d x \geq \frac{d^{\frac{2}{d}}}{d+2} \operatorname{vol}_{d-1}\left(\partial B_{2}^{d}\right)^{-\frac{2}{d}} \operatorname{vol}_{d}(K)^{\frac{d+2}{d}}
$$

Proof. Let $r(\xi)$ be the distance of the origin to the boundary of $K$ in direction $\xi$. By passing to spherical coordinates we get

$$
\left.\frac{1}{d} \int_{K} \sum_{i=1}^{d}\left|<x, e_{i}\right\rangle\right|^{2} d x=\frac{1}{d} \int_{\partial B_{2}^{d}} \int_{0}^{r(\xi)} \rho^{d+1} d \rho d \xi=\frac{1}{d(d+2)} \int_{\partial B_{2}^{d}} r(\xi)^{d+2} d \xi
$$

By Hölder's inequality we get that the above expression is greater than

$$
\frac{v l_{d-1}\left(\partial B_{2}^{d}\right)}{d(d+2)}\left(\frac{1}{\operatorname{vol}_{d-1}\left(\partial B_{2}^{d}\right)} \int_{\partial B_{2}^{d}} r(\xi)^{d} d \xi\right)^{\frac{d+2}{d}}=\frac{d^{\frac{2}{d}}}{d+2} \operatorname{vol}_{d-1}\left(\partial B_{2}^{d}\right)^{-\frac{2}{d}} \operatorname{vol}_{d}(K)^{\frac{d+2}{d}}
$$

The following lemma can be found in $[\mathrm{MP}]$. It is formulated there for the case 
Lemma 2.7. Let $K$ be a convex body in $\mathbb{R}^{d}$ such that the origin coincides with the center of gravity of $K$ and such that $K$ is in an isotropic position. Then we have

$$
B_{2}^{d}\left(c g(K), \frac{1}{24 e^{5} \sqrt{\pi}} \operatorname{vol}_{d}(K)^{\frac{1}{d}}\right) \subset K_{\frac{1}{4 e^{4}}} \operatorname{vol}_{d}(K)
$$

Proof. As in Lemma 2.3 let $\Theta(\xi)$ be the infimum of all numbers $t$ such that

$$
\operatorname{vol}_{d-1}(K \cap H(c g(K), \xi)) \geq e \operatorname{vol}_{d-1}(K \cap H(c g(K)+t \xi, \xi))
$$

By Lemma 2.3 we have

$$
\Theta(\xi) \geq \frac{1}{2 e^{3}} \frac{\operatorname{vol}_{d}(K)}{\operatorname{vol}_{d-1}(K \cap H(c g(K), \xi))}
$$

By Lemma 2.5 we get

$$
\Theta(\xi) \geq \frac{1}{2 e^{3} \sqrt{6} e^{\frac{3}{2}}}\left(\frac{1}{\operatorname{vol}_{d}(K)} \frac{1}{d} \int_{K} \sum_{i=1}^{d}\left|<x, e_{i}>\right|^{2} d x\right)^{\frac{1}{2}}
$$

We have

$$
\operatorname{vol}_{d}\left(B_{2}^{d}\right)=\frac{\pi^{\frac{d}{2}}}{\Gamma\left(\frac{d}{2}+1\right)} \leq \frac{\pi^{\frac{d-1}{2}}(2 e)^{\frac{d}{2}}}{d^{\frac{d+1}{2}}}
$$

and thus

$$
\operatorname{vol}_{d}\left(B_{2}^{d}\right)^{\frac{1}{d}} \leq \sqrt{\frac{2 \pi e}{d}}
$$

Therefore we get by Lemma 2.6

$$
\Theta(\xi) \geq \frac{1}{2 e^{3} \sqrt{6} e^{\frac{3}{2}}} \frac{d^{\frac{1}{d}}}{\sqrt{d+2}}\left(\frac{\operatorname{vol}_{d}(K)}{\operatorname{vol}_{d-1}\left(\partial B_{2}^{d}\right)}\right)^{\frac{1}{d}} \geq \frac{1}{12 e^{5} \sqrt{\pi}} \operatorname{vol}_{d}(K)^{\frac{1}{d}}
$$

On the other hand, we have

$$
\operatorname{vol}_{d}\left(K \cap H^{-}\left(c g(K)+\frac{\Theta(\xi)}{2} \xi, \xi\right)\right) \geq \int_{\frac{\Theta(\xi)}{2}}^{\Theta(\xi)} \operatorname{vol}_{d-1}(K \cap H(c g(K)+t \xi, \xi)) d t
$$

where $H^{-}\left(c g(K)+\frac{\Theta(\xi)}{2} \xi, \xi\right)$ is the half space not containing the origin. By the definition of $\Theta(\xi)$ this expression is greater than

$$
\frac{\Theta(\xi)}{2 e} \operatorname{vol}_{d-1}(K \cap H(c g(K), \xi))
$$

By Lemma 2.3 we get that this is greater than 
Therefore, every hyperplane that has distance

$$
\frac{1}{24 e^{5} \sqrt{\pi}} \operatorname{vol}_{d}(K)^{\frac{1}{d}}
$$

from the center of gravity cuts off a set of volume greater than $\frac{1}{4 e^{4}} \operatorname{vol}_{d}(K)$.

Proof of Theorem 2.1. We are choosing the vertices $x_{1}, \ldots, x_{n} \in \partial K$ of the polytope $P_{n} . N\left(x_{k}\right)$ denotes the normal to $\partial K$ at $x_{k} . x_{1}$ is chosen arbitrarily. Having chosen $x_{1}, \ldots, x_{k-1}$ we choose $x_{k}$ such that

$$
\left\{x_{1}, \ldots, x_{k-1}\right\} \cap \operatorname{Int}\left(K \cap H^{-}\left(x_{k}-\Delta_{k} N\left(x_{k}\right), N\left(x_{k}\right)\right)=\emptyset\right.
$$

where $\Delta_{k}$ is determined by

$$
\operatorname{vol}_{d}\left(K \cap H^{-}\left(x_{k}-\Delta_{k} N\left(x_{k}\right), N\left(x_{k}\right)\right)\right)=t
$$

It could be that the hyperplane $H\left(x_{k}-\Delta_{k} N\left(x_{k}\right), N\left(x_{k}\right)\right)$ is not tangential to the floating body $K_{t}$, but this does not affect the computation. We claim that this process terminates for some $n$ with

$$
n \leq e^{16 d} \frac{\operatorname{vol}_{d}\left(K \backslash K_{t}\right)}{t \operatorname{vol}_{d}\left(B_{2}^{d}\right)}
$$

This claim proves the theorem: If we cannot choose another $x_{n+1}$, then there is no cap of volume $t$ that does not contain an element of the polytope $P_{n}=\left[x_{1}, \ldots, x_{n}\right]$. By the theorem of Hahn-Banach we get $K_{t} \subset P_{n}$. We show now the claim. We put

$$
\begin{aligned}
& S_{n}=K \cap H^{-}\left(x_{n}-\Delta_{n} N\left(x_{n}\right), N\left(x_{n}\right)\right) \\
& S_{k}=K \cap\left(\bigcap_{i=k+1}^{n} H^{+}\left(x_{i}-\Delta_{i} N\left(x_{i}\right), N\left(x_{i}\right)\right)\right) \cap H^{-}\left(x_{k}-\Delta_{k} N\left(x_{k}\right), N\left(x_{k}\right)\right)
\end{aligned}
$$

for $k=1, \ldots, n-1$. We have for $k \neq l$ that

$$
\operatorname{vol}_{d}\left(S_{k} \cap S_{l}\right)=0
$$

Let $k<l<n$. Then we have

$$
\begin{aligned}
S_{k} \cap S_{l} & =K \cap\left(\bigcap_{i=k+1}^{n} H^{+}\left(x_{i}-\Delta_{i} N\left(x_{i}\right), N\left(x_{i}\right)\right)\right) \cap H^{-}\left(x_{k}-\Delta_{k} N\left(x_{k}\right), N\left(x_{k}\right)\right) \\
& \cap K \cap\left(\bigcap_{i=l+1}^{n} H^{+}\left(x_{i}-\Delta_{i} N\left(x_{i}\right), N\left(x_{i}\right)\right)\right) \cap H^{-}\left(x_{l}-\Delta_{l} N\left(x_{l}\right), N\left(x_{l}\right)\right) \\
& \subset H^{+}\left(x_{l}-\Delta_{l} N\left(x_{l}\right), N\left(x_{l}\right)\right) \cap H^{-}\left(x_{l}-\Delta_{l} N\left(x_{l}\right), N\left(x_{l}\right)\right)
\end{aligned}
$$


Thus we have

$$
\operatorname{vol}_{d}\left(S_{k} \cap S_{l}\right) \leq \operatorname{vol}_{d}\left(H\left(x_{l}-\Delta_{l} N\left(x_{l}\right), N\left(x_{l}\right)\right)\right)=0
$$

The case $k<l=n$ is shown in the same way. We have for $k=1, \ldots, n-1$

$$
\begin{aligned}
S_{k} & =K \cap\left(\bigcap_{i=k+1}^{n} H^{+}\left(x_{i}-\Delta_{i} N\left(x_{i}\right), N\left(x_{i}\right)\right)\right) \cap H^{-}\left(x_{k}-\Delta_{k} N\left(x_{k}\right), N\left(x_{k}\right)\right) \\
& \supset\left[x_{k}, K_{t}\right] \cap H^{-}\left(x_{k}-\Delta_{k} N\left(x_{k}\right), N\left(x_{k}\right)\right) \\
& \supset\left[x_{k},\left(K \cap H^{-}\left(x_{k}-\tilde{\Delta}_{k} N\left(x_{k}\right), N\left(x_{k}\right)\right)_{t}\right] \cap H^{-}\left(x_{k}-\Delta_{k} N\left(x_{k}\right), N\left(x_{k}\right)\right)\right.
\end{aligned}
$$

where $\tilde{\Delta}_{k}$ is determined by

$$
\operatorname{vol}_{d}\left(K \cap H^{-}\left(x_{k}-\tilde{\Delta}_{k} N\left(x_{k}\right), N\left(x_{k}\right)\right)\right)=4 e^{4} t
$$

By Lemma 2.7 there is an ellipsoid $\mathcal{E}$ contained in $\left(K \cap H^{-}\left(x_{k}-\tilde{\Delta}_{k} N\left(x_{k}\right), N\left(x_{k}\right)\right)\right)_{t}$ whose center is $c g\left(K \cap H^{-}\left(x_{k}-\tilde{\Delta}_{k} N\left(x_{k}\right), N\left(x_{k}\right)\right)\right)$ and that has volume

$$
\operatorname{vol}_{d}(\mathcal{E})=\frac{4 e^{4}}{\left(24 e^{5} \sqrt{\pi}\right)^{d}} t \operatorname{vol}_{d}\left(B_{2}^{d}\right)
$$

Since $\left(K \cap H^{-}\left(x_{k}-\tilde{\Delta}_{k} N\left(x_{k}\right), N\left(x_{k}\right)\right)\right)_{t}$ is contained in $K_{t}$, $\mathcal{E}$ is contained in $K_{t}$. Thus

$$
S_{k} \supset\left[x_{k}, \mathcal{E}\right] \cap H^{-}\left(x_{k}-\Delta_{k} N\left(x_{k}\right), N\left(x_{k}\right)\right)
$$

We claim now that $\left[x_{k}, \mathcal{E}\right] \cap H^{-}\left(x_{k}-\Delta_{k} N\left(x_{k}\right), N\left(x_{k}\right)\right)$ contains an ellipsoid $\tilde{\varepsilon}$ such that

$$
\operatorname{vol}_{d}(\tilde{\mathcal{E}})=\frac{4 e^{4}}{\left(24 e^{5} \sqrt{\pi}\right)^{d}} \frac{1}{\left(4 e^{5}\right)^{d}} t \operatorname{vol}_{d}\left(B_{2}^{d}\right)
$$

and consequently

$$
\operatorname{vol}_{d}\left(S_{k}\right) \geq \frac{4 e^{4}}{\left(24 e^{5} \sqrt{\pi}\right)^{d}} \frac{1}{\left(4 e^{5}\right)^{d}} t \operatorname{vol}_{d}\left(B_{2}^{d}\right)=\frac{4 e^{4}}{\left(96 e^{10} \sqrt{\pi}\right)^{d}} t \operatorname{vol}_{d}\left(B_{2}^{d}\right)
$$

For this we have to see that $\tilde{\Delta}_{k} \leq 4 e^{5} \Delta_{k}$. By the assumption $t \leq \frac{1}{4} e^{-5} \operatorname{vol}_{d}(K)$ we get that

$$
\operatorname{vol}_{d}\left(K \cap H^{-}\left(x_{k}-\tilde{\Delta}_{k} N\left(x_{k}\right), N\left(x_{k}\right)\right)\right) \leq \frac{1}{e} \operatorname{vol}_{d}(K)
$$

Therefore we get by $(2.1)$ that $c g(K) \in H^{+}\left(x_{k}-\tilde{\Delta}_{k} N\left(x_{k}\right), N\left(x_{k}\right)\right)$. We consider two cases. If 
then we have for all $t, \Delta_{k} \leq t \leq \tilde{\Delta}_{k}$, by the theorem of Brunn-Minkowski

$$
\begin{aligned}
\operatorname{vol}_{d-1}\left(K \cap H\left(c g(K), N\left(x_{k}\right)\right)\right. & \leq \operatorname{vol}_{d-1}\left(K \cap H\left(x_{k}-\tilde{\Delta}_{k} N\left(x_{k}\right), N\left(x_{k}\right)\right)\right) \\
& \leq \operatorname{vol}_{d-1}\left(K \cap H\left(x_{k}-t N\left(x_{k}\right), N\left(x_{k}\right)\right)\right)
\end{aligned}
$$

We get by $(2.2)$

$$
\Delta_{k} \geq \frac{t}{e \operatorname{vol}_{d-1}\left(K \cap H\left(c g(K), N\left(x_{k}\right)\right)\right)}
$$

By (2.8)

$$
\begin{aligned}
& \left(\tilde{\Delta}_{k}-\Delta_{k}\right) \operatorname{vol}_{d-1}\left(K \cap H\left(c g(K), N\left(x_{k}\right)\right)\right) \leq \\
& \quad \operatorname{vol}_{d}\left(K \cap H^{-}\left(x_{k}-\tilde{\Delta}_{k} N\left(x_{k}\right), N\left(x_{k}\right)\right)\right)-\operatorname{vol}_{d}\left(K \cap H^{-}\left(x_{k}-\Delta_{k} N\left(x_{k}\right), N\left(x_{k}\right)\right)\right)
\end{aligned}
$$

This implies

$$
\tilde{\Delta}_{k}-\Delta_{k} \leq \frac{\left(4 e^{4}-1\right) t}{\operatorname{vol}_{d-1}\left(K \cap H\left(c g(K), N\left(x_{k}\right)\right)\right)}
$$

Therefore we get

$$
\tilde{\Delta}_{k} \leq \frac{\left(4 e^{4}-1\right) t}{\operatorname{vol}_{d-1}\left(K \cap H\left(c g(K), N\left(x_{k}\right)\right)\right)}+\Delta_{k} \leq 4 e^{5} \Delta_{k}
$$

If

$$
\operatorname{vol}_{d-1}\left(K \cap H\left(x_{k}-\Delta_{k} N\left(x_{k}\right), N\left(x_{k}\right)\right)\right) \leq \operatorname{vol}_{d-1}\left(K \cap H\left(x_{k}-\tilde{\Delta}_{k} N\left(x_{k}\right), N\left(x_{k}\right)\right)\right)
$$

then by the theorem of Brunn-Minkowski we have for all $t, 0 \leq t \leq \Delta_{k}$, and all $s$, $\Delta_{k} \leq s \leq \tilde{\Delta}_{k}$

$$
\begin{aligned}
\operatorname{vol}_{d-1}\left(K \cap H\left(x_{k}-t N\left(x_{k}\right), N\left(x_{k}\right)\right)\right) & \leq \operatorname{vol}_{d-1}\left(K \cap H\left(x_{k}-\Delta_{k} N\left(x_{k}\right), N\left(x_{k}\right)\right)\right) \\
& \leq \operatorname{vol}_{d-1}\left(K \cap H\left(x_{k}-s N\left(x_{k}\right), N\left(x_{k}\right)\right)\right)
\end{aligned}
$$

We get

$$
\Delta_{k} \geq \frac{t}{\operatorname{vol}_{d-1}\left(K \cap H\left(x_{k}-\Delta_{k} N\left(x_{k}\right), N\left(x_{k}\right)\right)\right)}
$$

and

$$
\tilde{\Delta}_{k}-\Delta_{k} \leq \frac{\left(4 e^{4}-1\right) t}{\operatorname{vol}_{d-1}\left(K \cap H\left(x_{k}-\Delta_{k} N\left(x_{k}\right), N\left(x_{k}\right)\right)\right)}
$$

Therefore we get

$$
\tilde{\Delta}_{k} \leq \frac{\left(4 e^{4}-1\right) t}{\operatorname{vol}_{d-1}\left(K \cap H\left(x_{k}-\Delta_{k} N\left(x_{k}\right), N\left(x_{k}\right)\right)\right.}+\Delta_{k} \leq 4 e^{4} \Delta_{k}
$$

We have verified (2.7). From (2.6) and (2.7) we get

$$
\operatorname{vol}_{d}\left(K \backslash K_{t}\right) \geq \operatorname{vol}_{d}\left(\bigcup_{k=1}^{n} S_{k}\right)=\sum_{k=1}^{n} \operatorname{vol}_{d}\left(S_{k}\right) \geq n \frac{4 e^{4}}{\left(96 e^{10} \sqrt{\pi}\right)^{d}} t \operatorname{vol}_{d}\left(B_{2}^{d}\right)
$$

Thus we get (2.4)

$$
\operatorname{vol}_{d}\left(K \backslash K_{t}\right) \geq e^{-16 d} n t \operatorname{vol}_{d}\left(B_{2}^{d}\right)
$$




\section{The Illumination Body}

Theorem 3.1. Let $K$ be a convex body in $\mathbb{R}^{d}$ such that

$$
\frac{1}{c_{1}} B_{2}^{d} \subset K \subset c_{2} B_{2}^{d}
$$

Let $0 \leq t \leq\left(5 c_{1} c_{2}\right)^{-d-1} \operatorname{vol}_{d}(K)$ and let $n \in \mathbb{N}$ with

$$
\left(\frac{128}{7} \pi\right)^{\frac{d-1}{2}} \leq n \leq \frac{1}{32 e d t} \operatorname{vol}_{d}\left(K^{t} \backslash K\right)
$$

Then we have for every polytope $P_{n}$ that contains $K$ and has at most $n d-1$ dimensional faces

$$
\operatorname{vol}_{d}\left(K^{t} \backslash K\right) \leq 10^{7} d^{2}\left(c_{1} c_{2}\right)^{2+\frac{1}{d-1}} \operatorname{vol}_{d}\left(P_{n} \backslash K\right)
$$

We want to see what this result means for bodies with a smooth boundary. We have the asymptotic formula [W]

$$
\lim _{t \rightarrow 0} \frac{\operatorname{vol}_{d}\left(K^{t}\right)-\operatorname{vol}_{d}(K)}{t^{\frac{2}{d+1}}}=\frac{1}{2}\left(\frac{d(d+1)}{v l_{d-1}\left(B_{2}^{d-1}\right)}\right)^{\frac{2}{d+1}} \int_{\partial K} \kappa(x)^{\frac{1}{d+1}} d \mu(x)
$$

Thus we get

$$
\operatorname{vol}_{d}\left(K^{t}\right)-\operatorname{vol}_{d}(K) \sim t^{\frac{2}{d+1}} d \int_{\partial K} \kappa(x)^{\frac{1}{d+1}} d \mu(x)
$$

And by the theorem we have

$$
n \sim \frac{1}{d t} \operatorname{vol}_{d}\left(K^{t} \backslash K\right)
$$

Thus we get

$$
\operatorname{vol}_{d}\left(K^{t}\right)-\operatorname{vol}_{d}(K) \sim d\left(\frac{1}{d n} \operatorname{vol}_{d}\left(K^{t} \backslash K\right)\right)^{\frac{2}{d+1}} \int_{\partial K} \kappa(x)^{\frac{1}{d+1}} d \mu(x)
$$

$\mathrm{Or}$

$$
\begin{gathered}
\operatorname{vol}_{d}\left(K^{t} \backslash K\right)^{\frac{d-1}{d+1}} \sim d\left(\frac{1}{d n}\right)^{\frac{2}{d+1}} \int_{\partial K} \kappa(x)^{\frac{1}{d+1}} d \mu(x) \\
\operatorname{vol}_{d}\left(K^{t} \backslash K\right) \sim d\left(\frac{1}{n}\right)^{\frac{2}{d-1}}\left(\int_{\partial K} \kappa(x)^{\frac{1}{d+1}} d \mu(x)\right)^{\frac{d+1}{d-1}}
\end{gathered}
$$

By Theorem 3.1 we get now

$$
\operatorname{vol}_{d}\left(P_{n} \backslash K\right) \gtrsim \frac{1}{d}\left(\frac{1}{c_{1} c_{2}}\right)^{1+\frac{d}{d+1}}\left(\frac{1}{n}\right)^{\frac{2}{d-1}}\left(\int_{\partial K} \kappa(x)^{\frac{1}{d+1}} d \mu(x)\right)^{\frac{d+1}{d-1}}
$$

By a theorem of F. John $[\mathrm{J}]$ we have $c_{1} c_{2} \leq d$.

The following lemma is due to Bronshtein and Ivanov [BI] and Dudley $\left[D_{1}, D_{2}\right]$. It can also be found in [GRS]. 
Lemma 3.2. For all dimensions $d, d \geq 2$, and all natural numbers $n, n \geq 2 d$, there is a polytope $Q_{n}$ that has $n$ vertices and is contained in the Euclidean ball $B_{2}^{d}$ such that

$$
d_{H}\left(Q_{n}, B_{2}^{d}\right) \leq \frac{16}{7}\left(\frac{\operatorname{vol}_{d-1}\left(\partial B_{2}^{d}\right)}{\operatorname{vol}_{d-1}\left(B_{2}^{d-1}\right)}\right)^{\frac{2}{d-1}} n^{-\frac{2}{d-1}}
$$

We have that

$$
\begin{aligned}
\operatorname{vol}_{d-1}\left(\partial B_{2}^{d}\right) & =d \operatorname{vol}_{d}\left(B_{2}^{d}\right)=d \frac{\pi^{\frac{d}{2}}}{\Gamma\left(\frac{d}{2}+1\right)} \\
& =d \sqrt{\pi} \frac{\Gamma\left(\frac{d-1}{2}+1\right)}{\Gamma\left(\frac{d}{2}+1\right)} \operatorname{vol}_{d-1}\left(B_{2}^{d-1}\right) \leq d \sqrt{\pi} \operatorname{vol}_{d-1}\left(B_{2}^{d-1}\right)
\end{aligned}
$$

Since $d^{\frac{2}{d-1}} \leq 4$ and $(1-t)^{d} \geq 1-d t$ we get from (3.1)

$$
d_{H}\left(B_{2}^{d}, Q_{n}\right) \leq \frac{16}{7}\left(\frac{d \sqrt{\pi}}{n}\right)^{\frac{2}{d-1}} \leq \frac{64}{7} \pi n^{-\frac{2}{d-1}}
$$

Proof of Theorem 3.1. We denote the $d$-1-dimensional faces of $P_{n}$ by $F_{i}, i=$ $1, \ldots, n$, and the cones generated by the origin and a face $F_{i}$ by $C_{i}, i=1, \ldots, n$. Let $x_{i} \in F_{i}$ and $\xi_{i},\left\|\xi_{i}\right\|_{2}=1$, orthogonal to $F_{i}$ and pointing to the outside of $P_{n}$. Then $H\left(x_{i}, \xi_{i}\right)$ is the hyperplane containing $F_{i}$ and $H^{+}\left(x_{i}, \xi_{i}\right)$ the halfspace containing $P_{n}$. See figure 3.1. We may assume that the hyperplanes $H\left(x_{i}, \xi_{i}\right)$, $i=1, \ldots, n$, are supporting hyperplanes of $K$. Otherwise we can choose a polytope of lesser volume. Let $\Delta_{i}$ be the height of the set

$$
K^{t} \cap H^{-}\left(x_{i}, \xi_{i}\right) \cap C_{i}
$$

i.e. the smallest number $s$ such that

$$
K^{t} \cap H^{-}\left(x_{i}, \xi_{i}\right) \cap C_{i} \subset H^{+}\left(x_{i}+s \xi_{i}, \xi_{i}\right)
$$

Let $z_{i}$ be a point in $\partial K^{t} \cap C_{i}$ where the height $\Delta_{i}$ is attained. We may assume that $B_{2}^{d} \subset K \subset c B_{2}^{d}$ where $c=c_{1} c_{2}$. Also we may assume that

$$
P_{n} \subset 2 c B_{2}^{d}
$$

if we allow twice as many faces. This follows from (3.2): There is a polytope $Q_{k}$ such that $\frac{1}{2} B_{2}^{d} \subset Q_{k} \subset B_{2}^{d}$ and the number of vertices $k$ is smaller than $\left(\frac{128}{7} \pi\right)^{\frac{d-1}{2}}$. Thus $Q_{k}^{*}$ satisfies $B_{2}^{d} \subset Q_{k}^{*} \subset 2 B_{2}^{d}$ and has at most $\left(\frac{128}{7} \pi\right)^{\frac{d-1}{2}} d-1$-dimensional faces. As the new polytope $P_{n}$ we choose the intersection of $c Q_{k}^{*}$ with the original polytope $P_{n}$. Since we have by assumption that $\mathrm{n}$ is greater than $\left(\frac{128}{7} \pi\right)^{\frac{d-1}{2}}$ the new polytope has at most 
$d-1$-dimensional faces.

We show first that for $t$ with $0 \leq t \leq(5 c d)^{-d-1} v l_{d}(K)$ and all $i, i=1, \ldots, n$ we have

$$
\Delta_{i} \leq \frac{1}{d}
$$

Assume that there is a face $F_{i}$ with $\Delta_{i}>\frac{1}{d}$. Consider the smallest infinite cone $D_{i}$ having $z_{i}$ as vertex and containing $K$. Since $H\left(x_{i}, \xi_{i}\right)$ is a supporting hyperplane to $K$ and $K \subset c B_{2}^{d}$ we have

$$
K \subset D_{i} \cap H^{+}\left(x_{i}, \xi_{i}\right) \cap H^{-}\left(x_{i}-4 c \xi_{i}, \xi_{i}\right)
$$

and

$$
D_{i} \cap H^{-}\left(x_{i}, \xi\right)=\left[z_{i}, K\right] \cap H^{-}\left(x_{i}, \xi\right)
$$

We have

$$
\begin{gathered}
t=\operatorname{vol}_{d}\left(\left[z_{i}, K\right] \backslash K\right) \geq \operatorname{vol}_{d}\left(\left[z_{i}, K\right] \cap H^{-}\left(x_{i}, \xi_{i}\right)\right)=\operatorname{vol}_{d}\left(D_{i} \cap H^{-}\left(x_{i}, \xi_{i}\right)\right)= \\
\frac{1}{d} \Delta_{i} \operatorname{vol}_{d-1}\left(D_{i} \cap H\left(x_{i}, \xi_{i}\right)\right) \geq \frac{1}{d^{2}} \operatorname{vol}_{d-1}\left(D_{i} \cap H\left(x_{i}, \xi_{i}\right)\right)
\end{gathered}
$$

Thus

$$
\operatorname{vol}_{d-1}\left(D_{i} \cap H\left(x_{i}, \xi_{i}\right)\right) \leq d^{2} t
$$

Since (3.5) does not hold we have

$$
\begin{aligned}
\operatorname{vol}_{d-1}\left(D_{i} \cap H\left(x_{i}-4 c \xi_{i}, \xi_{i}\right)\right) & =\left(\frac{4 c+\Delta_{i}}{\Delta_{i}}\right)^{d-1} \operatorname{vol}_{d-1}\left(D_{i} \cap H\left(x_{i}, \xi_{i}\right)\right) \\
& \leq(4 c d+1)^{d-1} \operatorname{vol}_{d-1}\left(\left(D_{i} \cap H\left(x_{i}, \xi_{i}\right)\right)\right.
\end{aligned}
$$

By (3.6) we get

$$
\operatorname{vol}_{d-1}\left(D_{i} \cap H\left(x_{i}-4 c \xi_{i}, \xi_{i}\right)\right) \leq(4 c d+1)^{d-1} d^{2} t \leq(5 c d)^{d-1} d^{2} t
$$

Thus we get

$$
\begin{aligned}
\operatorname{vol}_{d}(K) & \leq \operatorname{vol}_{d}\left(D_{i} \cap H^{+}\left(x_{i}, \xi_{i}\right) \cap H^{-}\left(x_{i}-4 c \xi_{i}, \xi_{i}\right)\right) \\
& \leq 2 c(5 c d)^{d-1} d^{2} t \leq(5 c d)^{d+1} t
\end{aligned}
$$

Thus 
This is a contradiction to the assumption on $t$ in the hypothesis of the theorem. Thus we have shown (3.5). We consider now two cases: All those heights $\Delta_{i}$ that are smaller than $\frac{2 d t}{v_{d-1}\left(F_{i}\right)}$ and those that are greater. We may assume that $\Delta_{i}$, $i=1, \ldots, k$ are smaller than $\frac{2 d t}{v^{\prime} l_{d-1}\left(F_{i}\right)}$ and $\Delta_{i}, i=k+1, \ldots, n$ are strictly greater. We have

$$
\operatorname{vol}_{d}\left(\left(K^{t} \backslash P_{n}\right) \cap C_{i}\right)=\int_{0}^{\Delta_{i}} \operatorname{vol}_{d-1}\left(\left(K^{t} \backslash P_{n}\right) \cap C_{i} \cap H\left(x_{i}+s \xi_{i}, \xi_{i}\right)\right) d s
$$

Since $B_{2}^{d} \subset K \subset P_{n}$ we get

$$
\operatorname{vol}_{d}\left(\left(K^{t} \backslash P_{n}\right) \cap C_{i}\right) \leq \int_{0}^{\Delta_{i}} \operatorname{vol}_{d-1}\left(F_{i}\right)(1+s)^{d-1} d s \leq \Delta_{i}\left(1+\Delta_{i}\right)^{d-1} \operatorname{vol}_{d-1}\left(F_{i}\right)
$$

By (3.5) we get

$$
\operatorname{vol}_{d}\left(\left(K^{t} \backslash P_{n}\right) \cap C_{i}\right) \leq \Delta_{i}\left(1+\frac{1}{d}\right)^{d-1} \operatorname{vol}_{d-1}\left(F_{i}\right)
$$

For $i=1, \ldots, k$ we get

$$
\operatorname{vol}_{d}\left(\left(K^{t} \backslash P_{n}\right) \cap C_{i}\right) \leq \frac{2 d t}{\operatorname{vol}_{d-1}\left(F_{i}\right)}\left(1+\frac{1}{d}\right)^{d-1} \operatorname{vol}_{d-1}\left(F_{i}\right) \leq 2 e d t
$$

Thus we get

$$
\operatorname{vol}_{d}\left(\left(K^{t} \backslash P_{n}\right) \cap\left(\bigcup_{i=1}^{k} C_{i}\right)\right) \leq 2 k e d t \leq 2 n e d t
$$

By (3.4) we get

$$
\operatorname{vol}_{d}\left(\left(K^{t} \backslash P_{n}\right) \cap\left(\bigcup_{i=1}^{k} C_{i}\right)\right) \leq \frac{1}{8} \operatorname{vol}_{d}\left(K^{t} \backslash K\right)
$$

Now we consider the other faces. We have for $i=k+1, \ldots, n$

$$
\Delta_{i} \geq \frac{2 d t}{\operatorname{vol}_{d-1}\left(F_{i}\right)}
$$

We show that we have for $i=k+1, \ldots, n$

$$
\Delta_{i} \leq 5 c\left(\frac{5 c \operatorname{vol}_{d-1}\left(F_{i}\right)}{2 d \operatorname{vol}_{d}(K)}\right)^{\frac{1}{d-1}}
$$

Suppose that there is a face $F_{i}$ so that (3.9) does not hold. Then we have

$$
t=\operatorname{vol}_{d}\left(\left[z_{i}, K\right] \backslash K\right) \geq \operatorname{vol}_{d}\left(\left[z_{i}, K\right] \cap H^{-}\left(x_{i}, \xi_{i}\right)\right)=\frac{\Delta_{i}}{d} \operatorname{vol}_{d-1}\left(\left[z_{i}, K\right] \cap H\left(x_{i}, \xi_{i}\right)\right)
$$

Therefore we get by (3.8)

$$
\operatorname{vol}_{d-1}\left(\left[z_{i}, K\right] \cap H\left(x_{i}, \xi_{i}\right)\right)<\frac{d t}{-}<\frac{1}{-} \operatorname{vol}_{d-1}\left(F_{i}\right)
$$


By (3.3) we have that

$$
K \subset D_{i} \cap H^{+}\left(x_{i}, \xi_{i}\right) \cap H^{-}\left(x_{i}-4 c \xi_{i}, \xi_{i}\right)
$$

Thus

$$
\operatorname{vol}_{d}(K) \leq \operatorname{vol}_{d}\left(D_{i} \cap H^{-}\left(x_{i}-4 c \xi_{i}, \xi_{i}\right)\right)
$$

The cone $D_{i} \cap H^{-}\left(x_{i}-4 c \xi_{i}, \xi_{i}\right)$ has a height equal to $4 c+\Delta_{i}$. Therefore we get

$$
\operatorname{vol}_{d}(K) \leq \frac{1}{d}\left(4 c+\Delta_{i}\right)\left(\frac{4 c+\Delta_{i}}{\Delta_{i}}\right)^{d-1} \operatorname{vol}_{d-1}\left(D_{i} \cap H\left(x_{i}, \xi_{i}\right)\right)
$$

By (3.5) we have $\Delta_{i} \leq 1$. Therefore we get

$$
\begin{aligned}
\operatorname{vol}_{d}(K) & \leq \frac{5 c}{d}\left(\frac{5 c}{\Delta_{i}}\right)^{d-1} \operatorname{vol}_{d-1}\left(D_{i} \cap H\left(x_{i}, \xi_{i}\right)\right) \\
& =\frac{5 c}{d}\left(\frac{5 c}{\Delta_{i}}\right)^{d-1} \operatorname{vol}_{d-1}\left(\left[z_{i}, K\right] \cap H\left(x_{i}, \xi_{i}\right)\right)
\end{aligned}
$$

By (3.10) we get

$$
\operatorname{vol}_{d}(K) \leq \frac{5 c}{2 d}\left(\frac{5 c}{\Delta_{i}}\right)^{d-1} \operatorname{vol}_{d-1}\left(F_{i}\right)
$$

This inequality implies (3.9).

Let $y_{i}$ be the unique point

$$
y_{i}=\left[0, z_{i}\right] \cap H\left(x_{i}, \xi_{i}\right)
$$

We want to make sure that $y_{i} \in F_{i} \cap\left[z_{i}, K\right]$. This holds since $z_{i} \in C_{i} \cap H^{-}\left(x_{i}, \xi_{i}\right)$ and $\Delta_{i}>0$. Since $y_{i} \in F_{i}$ we have

$$
\operatorname{vol}_{d-1}\left(F_{i}\right)=\frac{\operatorname{vol}_{d-1}\left(B_{2}^{d-1}\right)}{\operatorname{vol}_{d-2}\left(\partial B_{2}^{d-1}\right)} \int_{\partial B_{2}^{d-1}} r_{i}(\eta)^{d-1} d \mu(\eta)
$$

where $r_{i}(\eta)$ is the distance of $y_{i}$ to the boundary $\partial F_{i}$ in direction $\eta, \eta \in \partial B_{2}^{d-1}$, and, since $y_{i} \in F_{i} \cap\left[z_{i}, K\right]$, we have

$$
\operatorname{vol}_{d-1}\left(F_{i} \cap\left[z_{i}, K\right]\right)=\frac{\operatorname{vol}_{d-1}\left(B_{2}^{d-1}\right)}{\operatorname{vol}_{d-2}\left(\partial B_{2}^{d-1}\right)} \int_{\partial B_{2}^{d-1}} \rho_{i}(\eta)^{d-1} d \mu(\eta)
$$

where $\rho_{i}(\eta)$ is the distance of $y_{i}$ to the boundary $\partial\left(F_{i} \cap\left[z_{i}, K\right]\right)$. Consider the set

$$
A_{i}=\left\{\eta \mid\left(1-\frac{1}{4 d}\right) r_{i}(\eta) \leq \rho_{i}(\eta)\right\}
$$

We show that

$$
\frac{1}{4} \operatorname{vol}_{d-1}\left(F_{i}\right) \leq \frac{\operatorname{vol}_{d-1}\left(B_{2}^{d-1}\right)}{1} \int r_{i}(\eta)^{d-1}-\rho_{i}(\eta)^{d-1} d \mu(\eta)
$$


We have

$$
\begin{aligned}
\frac{\operatorname{vol}_{d-1}\left(B_{2}^{d-1}\right)}{\operatorname{vol}_{d-2}\left(\partial B_{2}^{d-1}\right)} & \int_{A_{i}} r_{i}(\eta)^{d-1}-\rho_{i}(\eta)^{d-1} d \mu(\eta) \\
& \leq \frac{\operatorname{vol}_{d-1}\left(B_{2}^{d-1}\right)}{\operatorname{vol}_{d-2}\left(\partial B_{2}^{d-1}\right)} \int_{A_{i}} r_{i}(\eta)^{d-1}\left(1-\left(1-\frac{1}{4 d}\right)^{d-1}\right) d \mu(\eta) \\
& \leq \frac{1}{4} \frac{\operatorname{vol}_{d-1}\left(B_{2}^{d-1}\right)}{\operatorname{vol}_{d-2}\left(\partial B_{2}^{d-1}\right)} \int_{A_{i}} r_{i}(\eta)^{d-1} d \mu(\eta) \leq \frac{1}{4} \operatorname{vol}_{d-1}\left(F_{i}\right)
\end{aligned}
$$

Therefore we get

$$
\begin{aligned}
\frac{\operatorname{vol}_{d-1}\left(B_{2}^{d-1}\right)}{\operatorname{vol}_{d-2}\left(\partial B_{2}^{d-1}\right)} & \int_{A_{i}^{c}} r_{i}(\eta)^{d-1}-\rho_{i}(\eta)^{d-1} d \mu(\eta) \geq \\
& \frac{\operatorname{vol}_{d-1}\left(B_{2}^{d-1}\right)}{\operatorname{vol}_{d-2}\left(\partial B_{2}^{d-1}\right)} \int_{\partial B_{2}^{d-1}} r_{i}(\eta)^{d-1}-\rho_{i}(\eta)^{d-1} d \mu(\eta)- \\
& \frac{\operatorname{vol}_{d-1}\left(B_{2}^{d-1}\right)}{\operatorname{vol}_{d-2}\left(\partial B_{2}^{d-1}\right)} \int_{A_{i}} r_{i}(\eta)^{d-1}-\rho_{i}(\eta)^{d-1} d \mu(\eta) \geq \\
& \operatorname{vol}_{d-1}\left(F_{i}\right)-\operatorname{vol}_{d-1}\left(F_{i} \cap\left[z_{i}, K\right]\right)-\frac{1}{4} \operatorname{vol}_{d-1}\left(F_{i}\right)
\end{aligned}
$$

By (3.10) we get that this is greater than $\frac{1}{4} v o l_{d-1}\left(F_{i}\right)$. This implies

$$
\frac{1}{4} \operatorname{vol}_{d-1}\left(F_{i}\right) \leq \frac{\operatorname{vol}_{d-1}\left(B_{2}^{d-1}\right)}{\operatorname{vol}_{d-2}\left(\partial B_{2}^{d-1}\right)} \int_{A_{i}^{c}} r_{i}(\eta)^{d-1}-\rho_{i}(\eta)^{d-1} d \mu(\eta)
$$

Thus we have established (3.11).

We shall show that

$$
\operatorname{vol}_{d}\left(\left(K^{t} \backslash P_{n}\right) \cap C_{i}\right) \leq 20480 e d^{2} c^{2+\frac{1}{d-1}} \operatorname{vol}_{d}\left(\left(P_{n} \backslash K\right) \cap C_{i}\right)
$$

We have

$$
\operatorname{vol}_{d}\left(D_{i}^{c} \cap H^{+}\left(x_{i}, \xi_{i}\right) \cap C_{i}\right) \leq \operatorname{vol}_{d}\left(\left(P_{n} \backslash K\right) \cap C_{i}\right)
$$

Compare figure 3.2. Therefore, if we want to verify (3.12) it is enough to show

$$
\operatorname{vol}_{d}\left(\left(K^{t} \backslash P_{n}\right) \cap C_{i}\right) \leq 20480 e d^{2} c^{2+\frac{1}{d-1}} \operatorname{vol}_{d}\left(D_{i}^{c} \cap H^{+}\left(x_{i}, \xi_{i}\right) \cap C_{i}\right)
$$

We may assume that $y_{i}$ and $z_{i}$ are orthogonal to $H\left(x_{i}, \xi_{i}\right)$. This is accomplished by a linear, volume preserving map: Any vector orthogonal to $\xi_{i}$ is mapped onto 


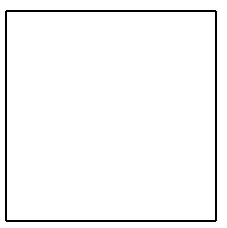

Let $w_{i}(\eta) \in D_{i}^{c} \cap H^{+}\left(x_{i}, \xi_{i}\right) \cap C_{i}$ such that $w_{i}(\eta)$ is an element of the 2-dimensional subspace containing $0, y_{i}$, and $y_{i}+\eta$. Let $\delta_{i}(\eta)$ be the distance of $w_{i}(\eta)$ to the plane $H\left(x_{i}, \xi_{i}\right)$. Then we have

$\frac{1}{d} \frac{\operatorname{vol}_{d-1}\left(B_{2}^{d-1}\right)}{\operatorname{vol}_{d-2}\left(\partial B_{2}^{d-1}\right)} \int_{A_{i}^{c}}\left(r_{i}(\eta)^{d-1}-\rho_{i}(\eta)^{d-1}\right) \delta_{i}(\eta) d \mu(\eta) \leq \operatorname{vol}_{d}\left(D_{i}^{c} \cap H^{+}\left(x_{i}, \xi_{i}\right) \cap C_{i}\right)$

Thus, in order to verify (3.12), it suffices to show

$$
\operatorname{vol}_{d}\left(\left(K^{t} \backslash P_{n}\right) \cap C_{i}\right) \leq
$$

$$
20480 e d^{2} c^{2+\frac{1}{d-1}} \frac{1}{d} \frac{\operatorname{vol}_{d-1}\left(B_{2}^{d-1}\right)}{\operatorname{vol}_{d-2}\left(\partial B_{2}^{d-1}\right)} \int_{A_{i}^{c}}\left(r_{i}(\eta)^{d-1}-\rho_{i}(\eta)^{d-1}\right) \delta_{i}(\eta) d \mu(\eta)
$$

In order to do this we shall show that for all $i=k+1, \ldots, n$ and all $\eta \in A_{i}^{c}$ there is $w_{i}(\eta)$ such that the distance $\delta_{i}(\eta)$ of $w_{i}$ from $H\left(x_{i}, \xi_{i}\right)$ satisfies

$$
\frac{\Delta_{i}}{\delta_{i}} \leq \begin{cases}32 d c & \text { if } \quad 0 \leq \alpha_{i} \leq \frac{\pi}{4} \\ \frac{160 d c^{2}}{r_{i}}\left(\frac{5 c \operatorname{vol}_{d-1}\left(F_{i}\right)}{2 d \operatorname{vol}_{d}(K)}\right)^{\frac{1}{d-1}} & \text { if } \quad \frac{\pi}{4} \leq \alpha_{i} \leq \frac{\pi}{2}\end{cases}
$$

The angles $\alpha_{i}(\eta)$ and $\beta_{i}(\eta)$ are given in figure 3.3. We have for all $\eta \in A_{i}^{c}$

$$
\begin{aligned}
\delta_{i} & =\left(r_{i}-\rho_{i}\right) \frac{\sin \left(\alpha_{i}\right) \sin \left(\beta_{i}\right)}{\sin \left(\pi-\alpha_{i}-\beta_{i}\right)} \quad 0 \leq \alpha_{i}, \beta_{i} \leq \frac{\pi}{2} \\
\Delta_{i} & =\rho_{i} \tan \alpha_{i}
\end{aligned}
$$

Thus we get

$$
\frac{\Delta_{i}}{\delta_{i}} \leq \frac{\rho_{i}}{r_{i}-\rho_{i}} \frac{\sin \left(\pi-\alpha_{i}-\beta_{i}\right)}{\cos \left(\alpha_{i}\right) \sin \left(\beta_{i}\right)} \leq \frac{\rho_{i}}{\left(r_{i}-\rho_{i}\right) \cos \left(\alpha_{i}\right) \sin \left(\beta_{i}\right)}
$$

By (3.11) we have $\rho_{i} \leq\left(1-\frac{1}{4 d}\right) r_{i}$. Therefore we get

$$
\frac{\Delta_{i}}{\delta_{i}} \leq \frac{4 d}{\cos \left(\alpha_{i}\right) \sin \left(\beta_{i}\right)}
$$

Since $B_{2}^{d} \subset K \subset P_{n} \subset 2 c B_{2}^{d}$ we get that $\tan \beta_{i} \geq \frac{1}{4 c}$ : Here we have to take into account that we applied a transform to $K$ mapping $y_{i}$ to $<\xi_{i}, y_{i}>\xi_{i}$. That leaves the distance of $F_{i}$ to the origin unchanged and $r_{i}(\eta)$ is less than $4 c$. If $\beta_{i} \geq \frac{\pi}{4}$ we have $\sin \beta_{i} \geq \frac{1}{\sqrt{2}}$. If $\beta_{i} \leq \frac{\pi}{4}$ then $\frac{1}{4 c} \leq \tan \beta_{i}=\frac{\sin \beta_{i}}{\cos \beta_{i}} \leq \sqrt{2} \sin \beta_{i}$. Therefore we get

$$
\underline{\Delta_{i}}<\underline{16 \sqrt{2} d c}
$$


Therefore we get for all $0 \leq \alpha_{i} \leq \frac{\pi}{4}$

$$
\frac{\Delta_{i}}{\delta_{i}} \leq 32 d c
$$

By (3.9) and (3.15) we get

$$
\frac{\Delta_{i}}{\delta_{i}} \leq \frac{1}{r_{i}-\rho_{i}} \frac{\sin \left(\pi-\alpha_{i}-\beta_{i}\right)}{\sin \left(\alpha_{i}\right) \sin \left(\beta_{i}\right)} 5 c\left(\frac{5 c \operatorname{vol}_{d-1}\left(F_{i}\right)}{2 d \operatorname{vol}_{d}(K)}\right)^{\frac{1}{d-1}}
$$

We proceed as in the estimate above and obtain

$$
\frac{\Delta_{i}}{\delta_{i}} \leq \frac{16 \sqrt{2} d c}{r_{i}} \frac{5 c}{\sin \left(\alpha_{i}\right)}\left(\frac{5 c \operatorname{vol}_{d-1}\left(F_{i}\right)}{2 d \operatorname{vol}_{d}(K)}\right)^{\frac{1}{d-1}}
$$

Thus we get for $\frac{\pi}{4} \leq \alpha_{i} \leq \frac{\pi}{2}$

$$
\frac{\Delta_{i}}{\delta_{i}} \leq \frac{32 d c}{r_{i}} 5 c\left(\frac{5 c \operatorname{vol}_{d-1}\left(F_{i}\right)}{2 d \operatorname{vol}_{d}(K)}\right)^{\frac{1}{d-1}}
$$

We verify now (3.13). By the definition of $A_{i}$ we get

$$
\begin{aligned}
\frac{\operatorname{vol}_{d-1}\left(B_{2}^{d-1}\right)}{v o l_{d-2}\left(\partial B_{2}^{d-1}\right)} \int_{A_{i}^{c}}\left(r_{i}(\eta)^{d-1}-\rho_{i}(\eta)^{d-1}\right) \delta_{i}(\eta) d \mu(\eta) \geq & \\
& \left(1-e^{-\frac{1}{8}}\right) \frac{v_{d-1}\left(B_{2}^{d-1}\right)}{v o l_{d-2}\left(\partial B_{2}^{d-1}\right)} \int_{A_{i}^{c}} r_{i}(\eta)^{d-1} \delta_{i} d \mu(\eta)
\end{aligned}
$$

We get by (3.15)

$$
\frac{1}{320 d c} \Delta_{i} \frac{\operatorname{vol}_{d-1}\left(B_{2}^{d-1}\right)}{\operatorname{vol}_{d-2}\left(\partial B_{2}^{d-1}\right)}\left\{\int_{\substack{A_{i}^{c} \\ \alpha_{i} \leq \frac{\pi}{4}}} r_{i}^{d-1} d \mu+\frac{1}{5 c}\left(\frac{2 d \operatorname{vol}_{d}(K)}{5 c \operatorname{vol}_{d-1}\left(F_{i}\right)}\right)^{\frac{1}{d-1}} \int_{\substack{A_{i}^{c} \\ \alpha_{i}>\frac{\pi}{4}}} r_{i}^{d} d \mu\right\}
$$

By (3.11) we get that either

$$
\frac{\operatorname{vol}_{d-1}\left(B_{2}^{d-1}\right)}{\operatorname{vol}_{d-2}\left(\partial B_{2}^{d-1}\right)} \int_{\substack{A_{i}^{c} \\ \alpha_{i} \leq \frac{\pi}{4}}} r_{i}^{d-1} d \mu \geq \frac{1}{8} \operatorname{vol}_{d-1}\left(F_{i}\right)
$$

or

$$
\frac{\operatorname{vol}_{d-1}\left(B_{2}^{d-1}\right)}{\operatorname{vol}_{d-2}\left(\partial B_{2}^{d-1}\right)} \int_{\substack{A_{i}^{c} \\ \alpha_{i}>\frac{\pi}{4}}} r_{i}^{d-1} d \mu \geq \frac{1}{8} \operatorname{vol}_{d-1}\left(F_{i}\right)
$$

In the first case we get for the above estimate

$$
\frac{\operatorname{vol}_{d-1}\left(B_{2}^{d-1}\right)}{\operatorname{vol}_{d-2}\left(\partial B_{2}^{d-1}\right)} \int_{A_{i}^{c}}\left(r_{i}(\eta)^{d-1}-\rho_{i}(\eta)^{d-1}\right) \delta_{i}(\eta) d \mu(\eta) \geq
$$

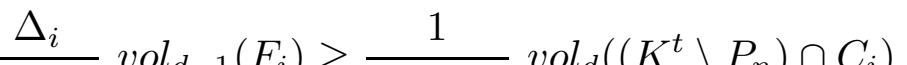


The last inequality is obtained by using (3.5): Since $B_{2}^{d} \subset K$ we have for all hyperplanes $H$ that are parallel to $F_{i} \operatorname{vol}_{d-1}\left(K^{t} \cap H \cap C_{i}\right) \leq\left(1+\Delta_{i}\right)^{d-1} \operatorname{vol}_{d-1}\left(F_{i}\right)$. By (3.5) we get $v l_{d-1}\left(K^{t} \cap H \cap C_{i}\right) \leq e \operatorname{vol}_{d-1}\left(F_{i}\right)$. In the second case we have

$$
\begin{aligned}
& \frac{\operatorname{vol}_{d-1}\left(B_{2}^{d-1}\right)}{\operatorname{vol}_{d-2}\left(\partial B_{2}^{d-1}\right)} \int_{A_{i}^{c}}\left(r_{i}(\eta)^{d-1}-\rho_{i}(\eta)^{d-1}\right) \delta_{i}(\eta) d \mu(\eta) \geq \\
& \frac{1}{5 c}\left(\frac{2 d \operatorname{vol}_{d}(K)}{5 c \operatorname{vol}_{d-1}\left(F_{i}\right)}\right)^{\frac{1}{d-1}} \frac{1}{320 d c} \Delta_{i} \frac{\operatorname{vol}_{d-1}\left(B_{2}^{d-1}\right)}{\operatorname{vol}_{d-2}\left(\partial B_{2}^{d-1}\right)} \int_{\alpha_{A_{i}}{ }_{i} \frac{\pi}{4}} r_{i}^{d} d \mu \geq \\
& \frac{1}{5 c}\left(\frac{2 d \operatorname{vol}_{d}(K)}{5 c \operatorname{vol}_{d-1}\left(F_{i}\right)}\right)^{\frac{1}{d-1}} \frac{1}{320 d c} \Delta_{i} \frac{\operatorname{vol}_{d-1}\left(B_{2}^{d-1}\right)}{\left(\operatorname{vol}_{d-2}\left(\partial B_{2}^{d-1}\right)\right)^{\frac{d}{d-1}}}\left(\int_{A_{i}^{c}>\frac{\pi}{4}} r_{i}^{d-1} d \mu\right)^{\frac{d}{d-1}} \geq \\
& \frac{1}{5 c}\left(\frac{2 d \operatorname{vol}_{d}(K)}{5 c \operatorname{vol}_{d-1}\left(F_{i}\right)}\right)^{\frac{1}{d-1}} \frac{\Delta_{i}}{320 d c} \operatorname{vol}_{d-1}\left(B_{2}^{d-1}\right)^{-\frac{1}{d-1}}\left(\frac{1}{8} \operatorname{vol}_{d-1}\left(F_{i}\right)\right)^{\frac{d}{d-1}}= \\
& \frac{1}{5 c}\left(\frac{d \operatorname{vol}_{d}(K)}{20 c \operatorname{vol}_{d-1}\left(B_{2}^{d-1}\right)}\right)^{\frac{1}{d-1}} \frac{\Delta_{i}}{2560 d c} \operatorname{vol}_{d-1}\left(F_{i}\right) \geq \\
& \frac{1}{5 c}\left(\frac{d \operatorname{vol}_{d}(K)}{20 c \operatorname{vol}_{d-1}\left(B_{2}^{d-1}\right)}\right)^{\frac{1}{d-1}} \frac{1}{2560 e d c} \operatorname{vol}_{d}\left(\left(K^{t} \backslash P_{n}\right) \cap C_{i}\right)
\end{aligned}
$$

Since $B_{2}^{d} \subset K$ we get

$$
\begin{aligned}
& \frac{\operatorname{vol}_{d-1}\left(B_{2}^{d-1}\right)}{\operatorname{vol}_{d-2}\left(\partial B_{2}^{d-1}\right)} \int_{A_{i}^{c}}\left(r_{i}(\eta)^{d-1}-\rho_{i}(\eta)^{d-1}\right) \delta_{i}(\eta) d \mu(\eta) \geq \\
& \frac{1}{5 c}\left(\frac{d \operatorname{vol}_{d}\left(B_{2}^{d}\right)}{20 c \operatorname{vol}_{d-1}\left(B_{2}^{d-1}\right)}\right)^{\frac{1}{d-1}} \frac{1}{2560 e d c} \operatorname{vol}_{d}\left(\left(K^{t} \backslash P_{n}\right) \cap C_{i}\right) \geq \\
& \frac{1}{5 c}\left(\frac{1}{20 c}\right)^{\frac{1}{d-1}} \frac{1}{2560 e d c} \operatorname{vol}_{d}\left(\left(K^{t} \backslash P_{n}\right) \cap C_{i}\right) \geq \\
&\left(20480 e d c^{2+\frac{1}{d-1}}\right)^{-1} \operatorname{vol}_{d}\left(\left(K^{t} \backslash P_{n}\right) \cap C_{i}\right)
\end{aligned}
$$

The second case gives a weaker estimate. Therefore we get for both cases

$$
\begin{aligned}
& \operatorname{vol}_{d}\left(\left(K^{t} \backslash P_{n}\right) \cap C_{i}\right) \leq \\
& \quad 20480 e d c^{2+\frac{1}{d-1}} \frac{\operatorname{vol}_{d-1}\left(B_{2}^{d-1}\right)}{\operatorname{vol}_{d-2}\left(\partial B_{2}^{d-1}\right)} \int_{A_{i}^{c}}\left(r_{i}(\eta)^{d-1}-\rho_{i}(\eta)^{d-1}\right) \delta_{i} d \mu(\eta)
\end{aligned}
$$

Thus we have verified (3.13) and by this also (3.12). By (3.12) we get

$$
\begin{aligned}
\operatorname{vol}_{d}\left(\left(K^{t} \backslash P_{n}\right) \cap\left(\bigcup_{i=k+1}^{n} C_{i}\right)\right) & \leq 20480 e d^{2} c^{2+\frac{1}{d-1}} \operatorname{vol}_{d}\left(\left(\bigcup_{i=k+1}^{n} C_{i}\right) \cap\left(P_{n} \backslash K\right)\right) \\
& \leq 20480 e d^{2} c^{2+\frac{1}{d-1}} \operatorname{vol}_{d}\left(\left(P_{n} \backslash K\right)\right)
\end{aligned}
$$

If the assertion of the theorem does not hold we have

$$
\operatorname{vol}_{d}\left(\left(P_{n} \backslash K\right)\right) \leq \frac{1}{2} \operatorname{vol}_{d}\left(K^{t} \backslash K\right)
$$


Thus we get

$$
\operatorname{vol}_{d}\left(\left(K^{t} \backslash P_{n}\right) \cap\left(\bigcup_{i=k+1}^{n} C_{i}\right)\right) \leq \frac{1}{8} \operatorname{vol}_{d}\left(K^{t} \backslash K\right)
$$

Together with (3.7) we obtain

$$
\operatorname{vol}_{d}\left(K^{t} \backslash P_{n}\right) \leq \frac{1}{4} \operatorname{vol}_{d}\left(K^{t} \backslash K\right) \leq \frac{1}{4}\left\{\operatorname{vol}_{d}\left(K^{t} \backslash P_{n}\right)+\operatorname{vol}_{d}\left(P_{n} \backslash K\right)\right\}
$$

By (3.17) we have

$$
\begin{aligned}
\operatorname{vol}_{d}\left(P_{n} \backslash K\right) & \leq \frac{1}{8} \frac{\operatorname{vol}_{d}\left(K^{t} \backslash K\right)}{20480 e d^{2} c^{2+\frac{1}{d-1}}} \\
& \leq \frac{1}{2} \operatorname{vol}_{d}\left(K^{t} \backslash K\right) \leq \frac{1}{2} \operatorname{vol}_{d}\left(K^{t} \backslash P_{n}\right)+\frac{1}{2} \operatorname{vol}_{d}\left(P_{n} \backslash K\right)
\end{aligned}
$$

This implies

$$
\operatorname{vol}_{d}\left(P_{n} \backslash K\right) \leq \operatorname{vol}_{d}\left(K^{t} \backslash P_{n}\right)
$$

Together with (3.18) we get now the contradiction

$$
\operatorname{vol}_{d}\left(K^{t} \backslash P_{n}\right) \leq \frac{1}{2} \operatorname{vol}_{d}\left(K^{t} \backslash P_{n}\right)
$$

\section{REFERENCES}

[B] K. Ball, Logarithmically concave functions and sections of convex sets in $\mathbb{R}^{n}$, Studia Mathematica 88 (1988), 69-84.

[BL] I. Bárány and D.G. Larman, Convex bodies, economic cap covering, random polytopes, Mathematika 35 (1988), 274-291.

[BI] E.M. Bronshtein and L.D. Ivanov, The approximation of convex sets by polyhedra, Siberian Mathematical Journal 16 (1975), 1110-1112.

$\left[\mathrm{D}_{1}\right] \quad \mathrm{R}$. Dudley, Metric entropy of some classes of sets with differentiable boundaries, Journal of Approximation Theory 10 (1974), 227-236.

$\left[\mathrm{D}_{2}\right] \quad$ R. Dudley, Correction to "Metric entropy of some classes of sets with differentiable boundaries", Journal of Approximation Theory 26 (1979), 192-193.

[F-T] L. FejesToth, Über zwei Maximumsaufgaben bei Polyedern, Tohoku Mathematical Journal 46 (1940), 79-83.

$\left[\mathrm{GMR}_{1}\right]$ Y. Gordon, M. Meyer, and S. Reisner, Volume approximation of convex bodies by polytopes - a constructive method, Studia Mathematica 111 (1994), 81-95.

$\left[\mathrm{GMR}_{2}\right]$ Y. Gordon, M. Meyer and S. Reisner, Constructing a polytope to approximate a convex body, Geometriae Dedicata 57 (1995), 217-222.

[GRS] Y. Gordon, S. Reisner, and C. Schütt, Umbrellas and polytopal approximation of the Euclidean ball, Journal of Approximation Theory.

[Gr 1 ] P.M. Gruber, Volume approximation of convex bodies by inscribed polytopes, Mathematische Annalen 281 (1988), 292-245.

[Gr 2 ] P.M. Gruber, Asymptotic estimates for best and stepwise approximation of convex bodies II, Forum Mathematicum 5 (1993), 521-538.

[GK] P.M. Gruber and P. Kenderov, Approximation of convex bodies by polytopes, Rend. Circolo Mat. Palermo 31 (1982), 195-225.

$[\mathrm{H}]$ D. Hensley, Slicing convex bodies-bounds for slice area in terms of the bodys covariance, 
$[\mathrm{J}] \quad$ F. John, Extremum problems with inequalities as subsidiary conditions, R. Courant Anniversary Volume, Interscience New York, 1948, pp. 187-204.

[Mac] A.M. Macbeath, An extremal property of the hypersphere, Proceedings of the Cambridge Philosophical Society 47 (1951), 245-247.

[MP] V. Milman and A. Pajor, Isotropic position and inertia ellipsoids and zonoids of the unit ball of a normed $n$-dimensional space, Geometric Aspects of Functional Analysis (J. Lindenstrauss and V.D. Milman, eds.), Israel Seminar(GAFA) 1987-88, Springer-Verlag, 1989, pp. 64-104.

[Mü] J.S. Müller, Approximation of the ball by random polytopes, Journal of Approximation Theory 63 (1990), 198-209.

[R] C.A. Rogers, Packing and Covering, Cambridge University Press, 1964.

[S] C. Schütt, The convex floating body and polyhedral approximation, Israel Journal of Mathematics 73 (1991), 65-77.

[SW] C. Schütt and E. Werner, The convex floating body, Mathematica Scandinavica 66 (1990), 275-290.

[W] E. Werner, Illumination Bodies and the Affine Surface Area, Studia Mathematica 110 (1994), 257-269.

Mathematisches Seminar, Christian Albrechts Universität, D-24098 Kiel, GerMANY

Department of Mathematics, Oklahoma State University, Stillwater, Oklahoma 74078 


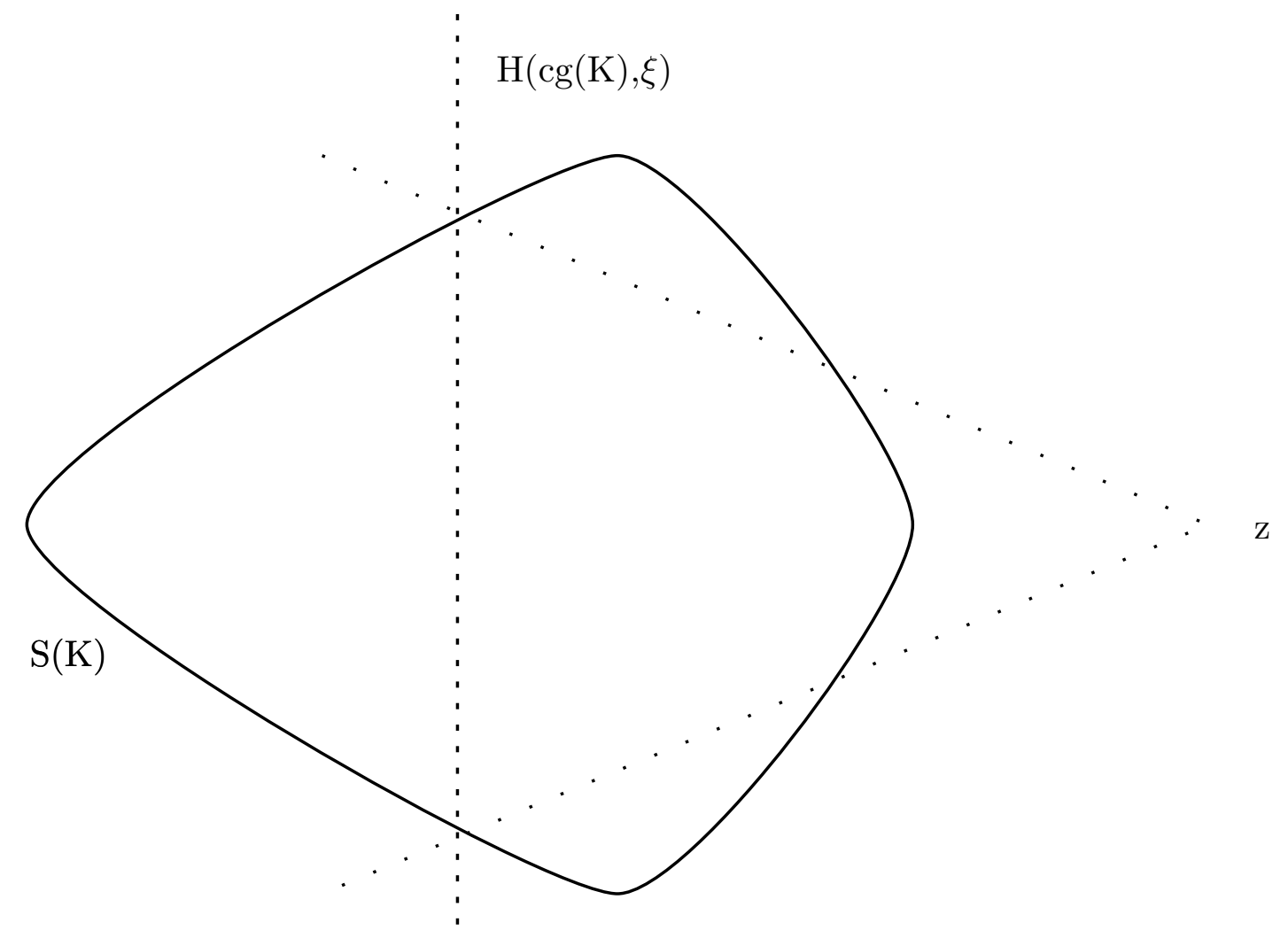

Figure 2.1 


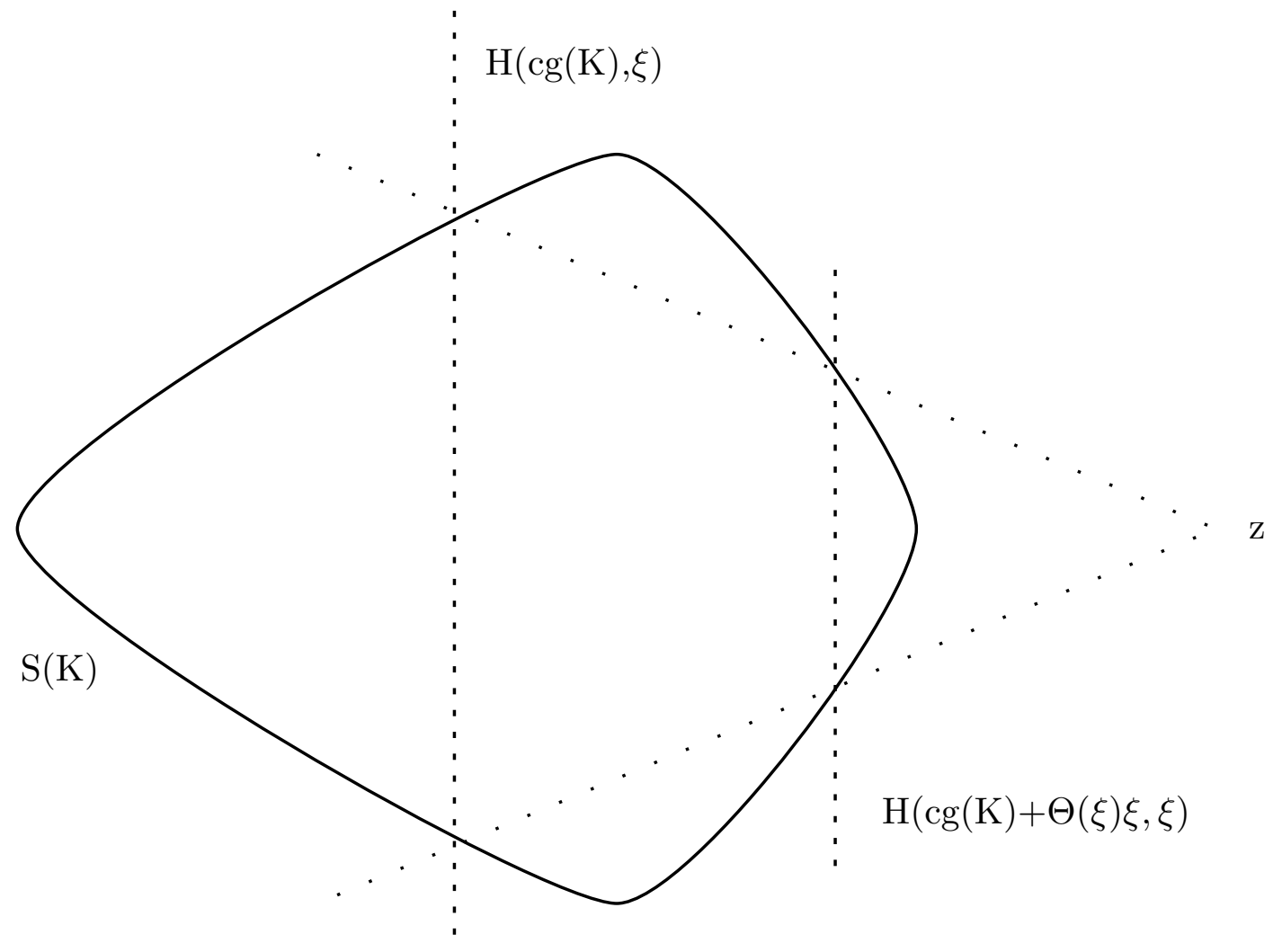

Figure 2.2 


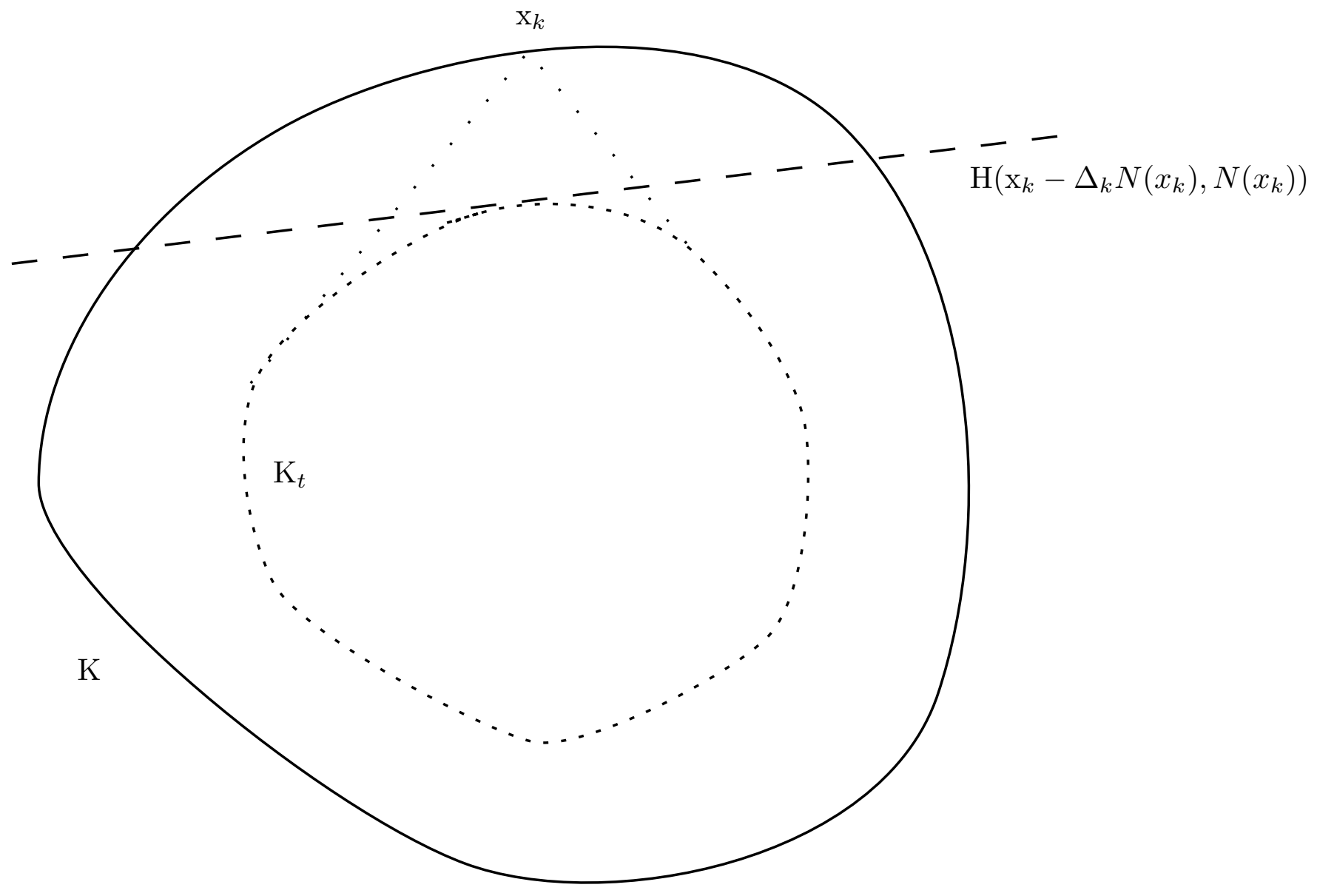

Figure 2.3 


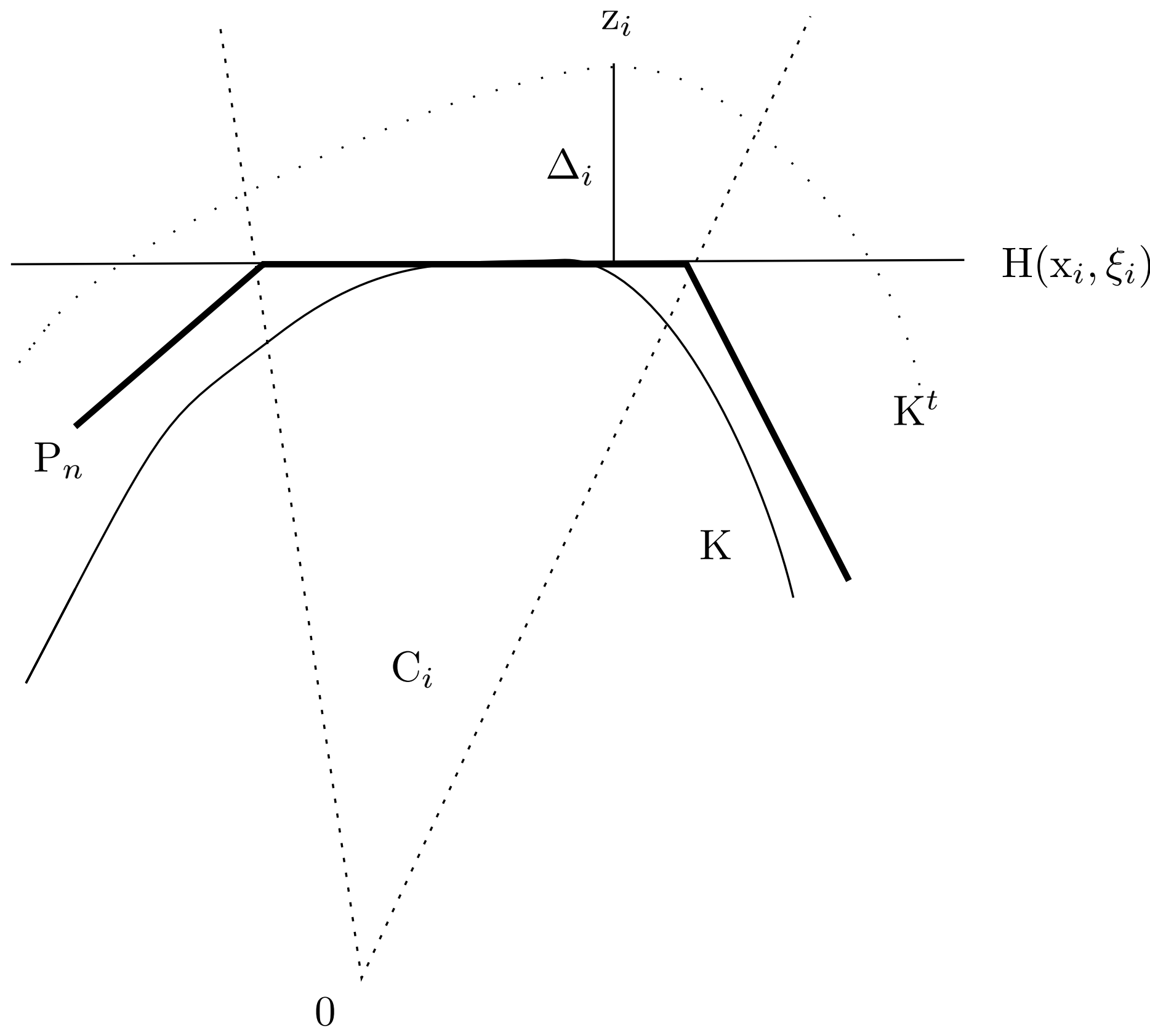

Figure 3.1 


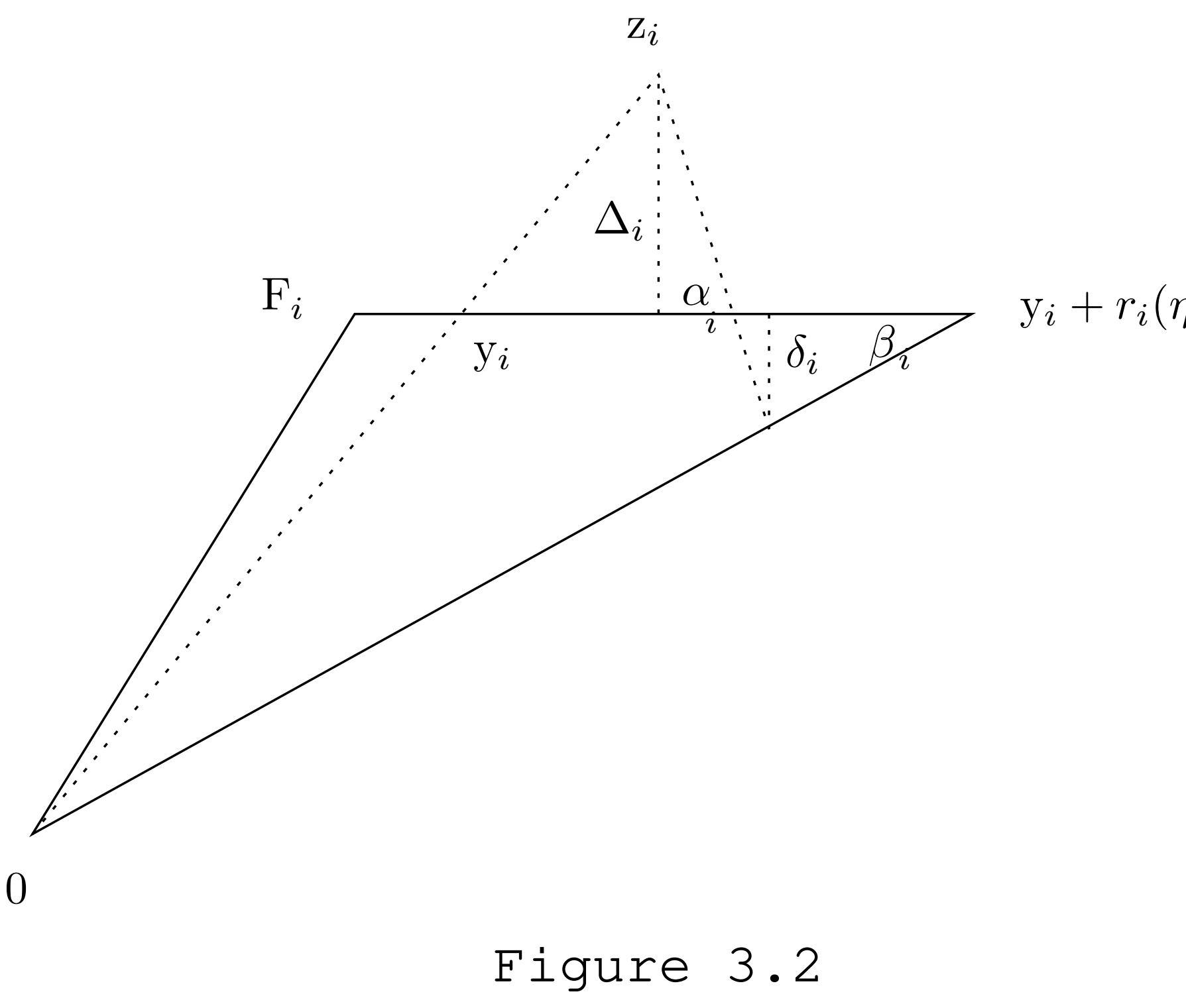




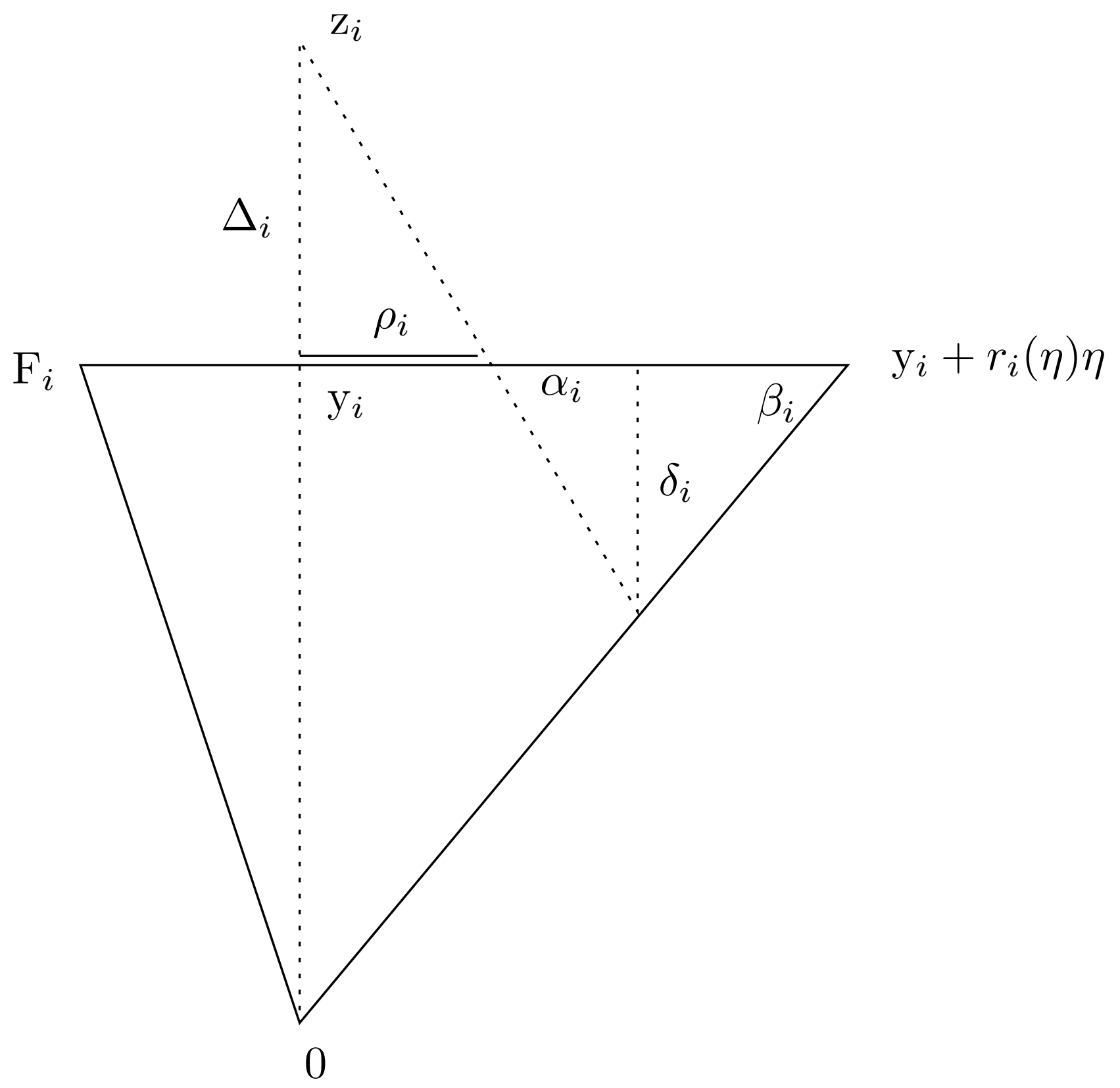

Figure 3.3 